\title{
Discrete conformal maps and ideal hyperbolic polyhedra
}

\author{
ALEXANDER I BOBENKO \\ ULRICH PINKALL \\ BORIS A SPRINGBORN
}

\begin{abstract}
We establish a connection between two previously unrelated topics: a particular discrete version of conformal geometry for triangulated surfaces, and the geometry of ideal polyhedra in hyperbolic three-space. Two triangulated surfaces are considered discretely conformally equivalent if the edge lengths are related by scale factors associated with the vertices. This simple definition leads to a surprisingly rich theory featuring Möbius invariance, the definition of discrete conformal maps as circumcirclepreserving piecewise projective maps, and two variational principles. We show how literally the same theory can be reinterpreted to address the problem of constructing an ideal hyperbolic polyhedron with prescribed intrinsic metric. This synthesis enables us to derive a companion theory of discrete conformal maps for hyperbolic triangulations. It also shows how the definitions of discrete conformality considered here are closely related to the established definition of discrete conformality in terms of circle packings.
\end{abstract}

$52 \mathrm{C} 26 ; 52 \mathrm{~B} 10,57 \mathrm{M} 50$

\section{Introduction}

Recall that two Riemannian metrics $g$ and $\tilde{g}$ on a smooth manifold $M$ are called conformally equivalent if

$$
\tilde{g}=e^{2 u} g
$$

for a function $u \in C^{\infty}(M)$. In the discrete theory that we consider here, smooth manifolds are replaced with triangulated piecewise euclidean manifolds, and the discrete version of a conformal change of metric is to multiply all edge lengths with scale factors that are associated with the vertices (Definition 2.1.1). Apparently, the idea to model conformal transformations in a discrete setting by attaching scale factors to the vertices appeared first in the four-dimensional Lorentz-geometric context of the Regge calculus; see Roček and Williams [37]. The Riemann-geometric version of this notion appeared in Luo's work on "combinatorial Yamabe flow" [24]. He showed that this flow is the gradient flow of a locally convex function. Later, an explicit formula for this 
function was found ( $E_{\mathrm{T}, \Theta, \lambda}$ defined in equation (4-1), with $\Theta=0$ ), and this lead to an efficient numerical method to compute discrete conformal maps, suitable for applications in computer graphics; see the second and third authors and Schröder [40]. (Some basic theory of conformal equivalence and conformal maps in Section 2 and the first variational principle in Section 4 are already covered or at least touched upon in this earlier paper.) The variational principles described in Section 4 reduce the discrete conformal mapping problems described in Section 3 to problems of convex optimization. Figures 1 and 2 show examples of discrete conformal maps that were obtained this way.
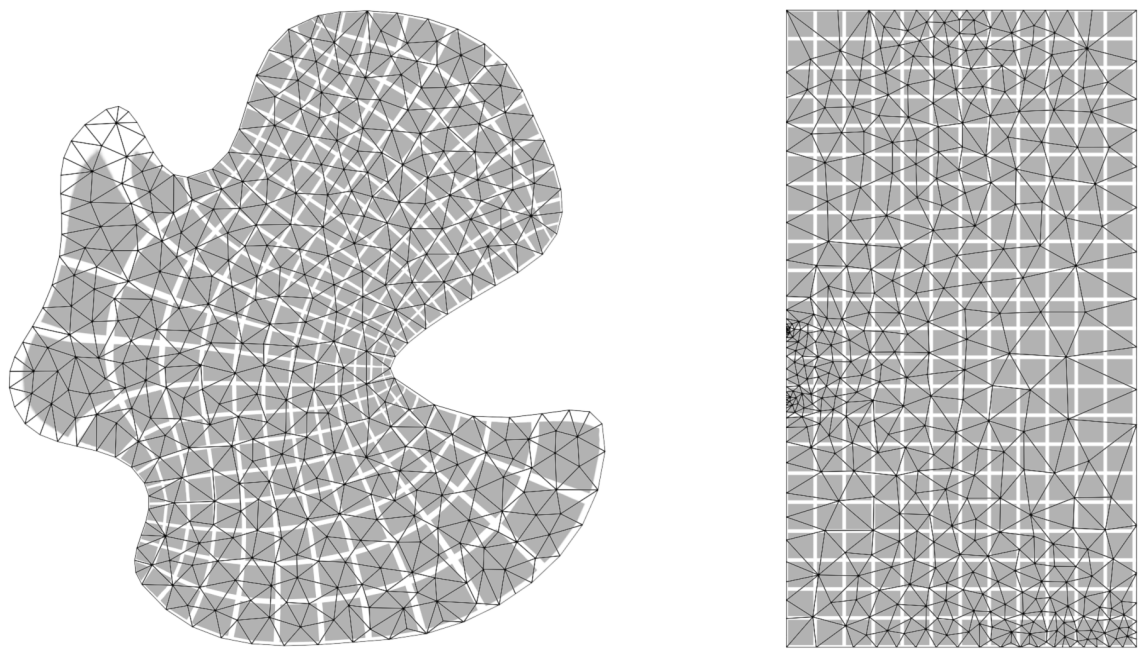

Figure 1: Discrete conformal map to a rectangle

Möbius transformations preserve the discrete conformal class (Section 2.5), and this makes it possible to construct discrete conformal maps to regions bounded by circular polygons (Section 3.3), discrete analogs of the classical Riemann maps.

The first variational principle (Section 4.1) involves a function of the (logarithmic) scale factors $u$. The second variational principle (Section 4.3) involves a function of the triangle angles. The two variational principles are Legendre duals in a precise way, but we do not dwell on this point. The corresponding variational principles of the classical smooth theory are discussed in Appendix B.

There are clear signs in Sections 2 and 4 that indicate a connection with hyperbolic geometry: the appearance of Milnor's Lobachevsky function $Л(x)$, the fact that the second variational principle is almost the same as Rivin's variational principle for ideal hyperbolic polyhedra with prescribed dihedral angles [34], and the definition 

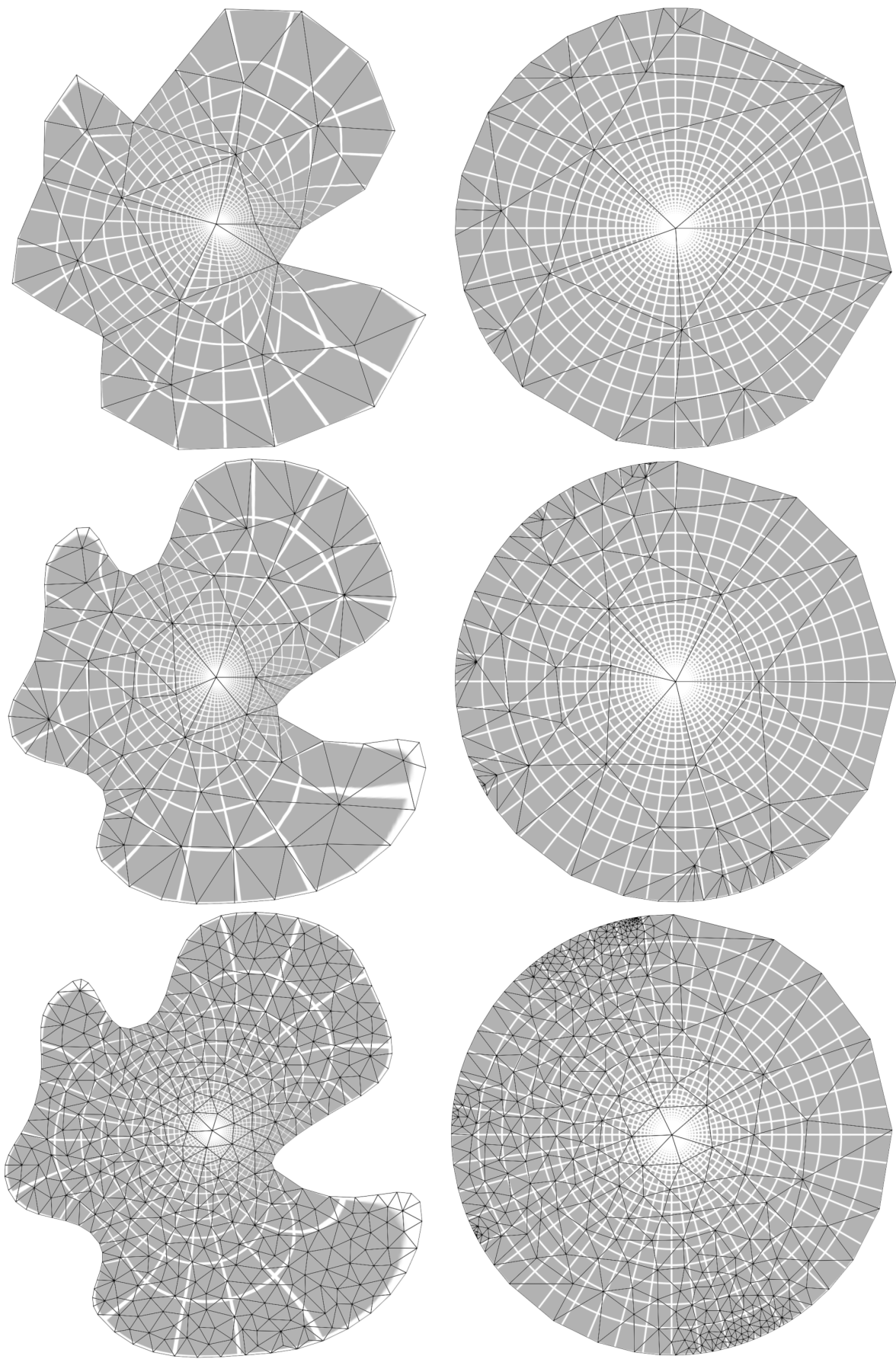

Figure 2: Discrete Riemann maps 
of discrete conformal maps in terms of circumcircle-preserving piecewise projective functions (Section 2.6). This connection with two- and three-dimensional hyperbolic geometry is the topic of Section 5. Reversing a construction of Penner [32] and Epstein and Penner [11], we equip a triangulated piecewise euclidean surface with a canonical hyperbolic metric with cusps. Discrete conformal maps are precisely the isometries with respect to this hyperbolic metric (Section 5.1). The logarithmic edge lengths $\lambda$ (Section 2.1) and the length-cross-ratios that characterize a discrete conformal class (Section 2.3) are Penner coordinates and shear coordinates, respectively, of the corresponding hyperbolic surface (Section 5.3). The problem of flattening a triangulation discretely conformally is equivalent to constructing an ideal hyperbolic polyhedron with prescribed intrinsic metric (Section 5.4). With this interpretation of discrete conformality in terms of three-dimensional hyperbolic geometry, the two variational principles of Section 4 are seen to derive from Schläfli's differential volume formula and Milnor's equation for the volume of an ideal tetrahedron (Section 5.5).

Once this connection between discrete conformality and hyperbolic polyhedra is established, it is straightforward to obtain a modified version of discrete conformality that pertains to triangulations composed of hyperbolic triangles instead of euclidean ones (Section 6). This is the theory of discrete conformal uniformization of triangulated higher-genus surfaces over the hyperbolic plane. It has been applied, for example, for the hyperbolization of euclidean ornaments; see von Gagern and Richter-Gebert [14]. (It is equally straightforward to obtain a corresponding theory for spherical triangulations, but the functions involved in the corresponding variational principles are not convex. We do not pursue this branch of the theory here.)

The connection with hyperbolic polyhedra entrains a connection between the discrete notion of conformality considered here and circle patterns, another discretization of the same concept. Thurston introduced patterns of circles as an elementary geometric visualization of hyperbolic polyhedra [42, Chapter 13]. He rediscovered Koebe's circle packing theorem [21] and showed that it followed from Andreev's work on hyperbolic polyhedra [2; 3]; see also Roeder, Hubbard and Dunbar [39]. Thurston's conjecture that circle packings could be used to approximate the classical Riemann map, which was later proved by Rodin and Sullivan [38], set off a flurry of research that led to a full-fledged theory of discrete analytic functions and conformal maps based on packings and patterns of circles; see Stephenson [41]. (The circle packing version of Luo's "combinatorial Yamabe flow" is the "combinatorial Ricci flow" of Chow and Luo [7] and Gu and Yau [16].) The relationship between these two theories of discrete conformality is now clear: the circle packing theory deals with hyperbolic polyhedra with prescribed dihedral angles and the notion of discrete conformality considered 
here deals with hyperbolic polyhedra with prescribed metric. In Appendix C.1 we discuss the relationship between the variational principles for discrete conformal maps (Section 4) and two variational principles for circle patterns. One is due to Rivin [34] (see also the recent survey article by Futer and Guéritaud [13], which provides a wealth of material that is otherwise difficult to find), and the other is again related to it by the same sort of singular Legendre duality; see the first and third authors [6]. Variational principles for circle patterns are important in discrete differential geometry in particular for constructing discrete minimal surfaces; see the first and third authors and Hoffmann [4]. Instead of triangulations one can consider meshes composed of polygons that are inscribed in circles (Section C.2), and we consider the problem to map multiply connected domains to domains bounded by polygons inscribed in circles, a discrete version of circle domains (Section C.3).

Two important questions are not addressed in this paper. The first is the question of convergence. Of course we do believe (under not too restrictive assumptions that have yet to be worked out) that discrete conformal maps approximate conformal maps if the triangulation is fine enough. Figure 2 clearly suggests that a version of the Rodin-Sullivan theorem [38] also holds in this case. But all this has yet to be proved.

The other question concerns the solvability of the discrete conformal mapping problems of Section 3. A solution may not exist due to violated triangle inequalities. Fairly obvious necessary conditions and how they relate to properties of the function $E_{\mathrm{T}, \Theta, \lambda}$ appearing in the first variational principle are discussed in Appendix A. In the numerous numerical experiments that we have made, we have observed that a solution exists if the necessary conditions are satisfied, no triangles are almost degenerate to begin with, and the triangulation is not too coarse. But to find necessary and sufficient conditions for solvability seems to be an intractable problem in this setting. After all, this would amount to giving necessary and sufficient conditions for the existence of a (not necessarily convex) ideal hyperbolic polyhedron with prescribed intrinsic metric and prescribed combinatorial type. The way out is to restrict oneself to convex polyhedra while widening the concept of discrete conformal map to allow for combinatorial changes (Section 5.1). Rivin proved that any hyperbolic metric with cusps on the sphere is realized by a unique ideal polyhedron [35]. This translates into an existence statement for discrete conformal maps. (Conversely, this suggests a variational proof of Rivin's theorem very similar to the recent constructive proof by the first author and Izmestiev [5] of Alexandrov's classical polyhedral realization theorem [1].)

Previous versions of this article have been available as the preprint arXiv:1005.2698 since May 2010. For the published version, the text has been restructured according to the suggestions of the referee. The mathematical content has not changed. 


\section{Discrete conformal equivalence and maps}

\subsection{Discrete conformal equivalence}

A surface is a connected 2-dimensional manifold, possibly with boundary. A surface triangulation, or triangulation for short, is a surface that is a CW-complex whose faces (2-cells) are triangles which are glued edge-to-edge. We will denote the sets of vertices ( 0 -cells), edges (1-cells), and faces of a triangulation $\mathrm{T}$ by $V_{\mathrm{T}}, E_{\mathrm{T}}$, and $T_{\mathrm{T}}$, and we will often drop the subscript $T$ if the triangulation is clear from the context. We will also write $A_{\top}$ for the set of triangle angles, where angles means corners, or triangle-vertex incidences, not angle measures.

A euclidean surface triangulation, or euclidean triangulation for short, is a surface triangulation equipped with a metric so that $\mathrm{T} \backslash V_{\mathrm{T}}$ is locally isometric to the euclidean plane, or half-plane if there is boundary, and the edges are geodesic segments. In other words, a euclidean surface triangulation is a surface consisting of euclidean triangles that are glued edge-to-edge. At the vertices, the metric may have cone-like singularities.

A euclidean triangulation is uniquely determined by a triangulation $T$ and a function $\ell: E_{\mathrm{T}} \rightarrow \mathbb{R}_{>0}$ assigning a length to every edge in such a way that the triangle inequalities are satisfied for every triangle in $T_{\mathrm{T}}$. We call such a positive function $\ell$ on the edges that satisfies all triangle inequalities a discrete metric on $\mathrm{T}$, and we denote the resulting euclidean triangulation by $(T, \ell)$.

In this paper, we will assume for simplicity that the triangulations are simplicial complexes. This means that a triangle may not be glued to itself at a vertex or along an edge, and the intersection of two triangles is either empty or it consists of one vertex or one edge. This restrictions to simplicial complexes allows us to use simple notation: we will denote by $i j$ the edge with vertices $i$ and $j$, by $i j k$ the triangle with vertices $i, j$, and $k$, and by ${ }_{j k}^{i}$ the corner at vertex $i$ in triangle $i j k$. If $f, g, h$, and $\phi$ are functions on $V, E, T$, and $A$, respectively, we will write $f_{i}, g_{i j}, h_{i j k}$, and $\phi_{j k}^{i}$ for $f(i), g(i j), h(i j k)$, and $\phi\left(\begin{array}{c}i \\ j k\end{array}\right)$. But while this restriction to simplicial complexes is notationally very convenient, it is a priori uncalled for. There are a few exceptions, like Sections 3.2 and 3.3 on mapping to the sphere and disk, but in general the domain of validity of the theory presented here extends beyond the simplicial case.

The vector spaces of real-valued functions on the sets of vertices, edges and angles will be denoted by $\mathbb{R}^{V}, \mathbb{R}^{E}$ and $\mathbb{R}^{A}$, respectively.

Definition 2.1.1 (Luo [24]) Two combinatorially equivalent euclidean triangulations, $(\mathrm{T}, \ell)$ and $(\mathrm{T}, \tilde{\ell})$, are discretely conformally equivalent if the discrete metrics $\ell$ and $\tilde{\ell}$ 
are related by

$$
\tilde{\ell}_{i j}=e^{\left(u_{i}+u_{j}\right) / 2} \ell_{i j}
$$

for some $u \in \mathbb{R}^{V}$. This defines an equivalence relation on the set of discrete metrics on $\mathrm{T}$, of which an equivalence class is called a discrete conformal class of discrete metrics, or a discrete conformal structure, on $\mathrm{T}$.

Instead of the edge lengths $\ell$ we will often use the logarithmic lengths

$$
\lambda=2 \log \ell .
$$

(The reason for the factor of 2 will become apparent in Section 5.) In terms of these logarithmic lengths, relation $(2-1)$ between $\ell$ and $\tilde{\ell}$ becomes linear:

$$
\tilde{\lambda}_{i j}=\lambda_{i j}+u_{i}+u_{j}
$$

Remark 2.1.2 (Dimension of "discrete Teichmüller space") The set of all discrete metrics on a triangulation $T$ is a manifold whose dimension is the number of edges, $|E|$. This manifold of metrics is fibered by the discrete conformal classes, each of which is a submanifold of dimension $|V|$, the number of vertices. The corresponding "discrete Teichmüller space", ie the manifold of discrete conformal classes, has dimension $|E|-|V|$. If $\mathrm{T}$ triangulates a closed surface of genus $g$, one obtains $|E|-|V|=$ $6 g-6+2|V|$, which is also the dimension of $\mathcal{T}_{g,|V|}$, the Teichmüller space of a genus- $g$ Riemann surfaces with $|V|$ punctures. This is no coincidence. The discrete conformal classes actually correspond to points in the Teichmüller space $\mathcal{T}_{g,|V|}$ (see Section 5.1).

\subsection{The two most simple cases}

(1) If the triangulation $\mathrm{T}$ consists of a single triangle $i j k$, then any two euclidean triangulations $(\mathrm{T}, \ell)$ and $(\mathrm{T}, \tilde{\ell})$ are discretely conformally equivalent, because the three equations

$$
\tilde{\ell}_{i j}=e^{\left(u_{i}+u_{j}\right) / 2} \ell_{i j}, \quad \tilde{\ell}_{j k}=e^{\left(u_{j}+u_{k}\right) / 2} \ell_{j k}, \quad \tilde{\ell}_{k i}=e^{\left(u_{k}+u_{i}\right) / 2} \ell_{k i}
$$

always have a unique solution for $u_{i}, u_{j}$ and $u_{k}$ :

$$
e^{u_{i}}=\frac{\tilde{\ell}_{i j} \ell_{j k} \tilde{\ell}_{k i}}{\ell_{i j} \tilde{\ell}_{j k} \ell_{k i}}, \ldots
$$

(2) Now let $\mathrm{T}$ be the triangulation consisting of two triangles $i j k$ and $i l j$ glued along edge $i j$ as shown in Figure 3, and let $\ell$ and $\tilde{\ell}$ be two discrete metrics on $\mathrm{T}$. 


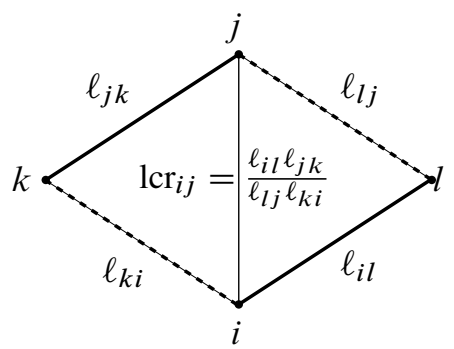

Figure 3: The length-cross-ratio on edge $i j$ : the lengths of the bold solid and bold dashed edges appear in the numerator and denominator, respectively.

What is the condition for $(\mathrm{T}, \ell)$ and $(\mathrm{T}, \tilde{\ell})$ to be discretely conformally equivalent? For each triangle considered separately, the corresponding equations (2-1) determine unique solutions for the values of $u$ on its vertices. For each of the common vertices $i$ and $j$ one obtains two values and the necessary and sufficient condition for discrete conformal equivalence is that they are equal, which is equivalent to the condition

$$
\frac{\ell_{i l} \ell_{j k}}{\ell_{l j} \ell_{k i}}=\frac{\tilde{\ell}_{i l} \tilde{\ell}_{j k}}{\tilde{\ell}_{l j} \tilde{\ell}_{k i}} .
$$

\subsection{Length-cross-ratios}

The simple reasoning of Section 2.2 extends to the general case: let $\mathrm{T}$ be any triangulation, and let $\ell$ and $\tilde{\ell}$ be two discrete metrics on $\mathrm{T}$. For each triangle $i j k$ of $\mathrm{T}$, considered separately, equations (2-1) determine unique values for $u$. Thus, for each vertex $i \in V$, one obtains one value for $u_{i}$ per adjacent triangle. These values are in general different. They agree for each vertex if and only if the discrete metrics $\ell$ and $\tilde{\ell}$ are discretely conformally equivalent. Since the vertex links are connected, it suffices to consider values obtained from adjacent triangles. This leads to Proposition 2.3.2 below, where the condition for discrete conformal equivalence is given in terms of the so-called length-cross-ratios:

Definition 2.3.1 For each interior edge $i j$ between triangles $i j k$ and $i l j$ as in Figure 3, define the length-cross-ratio induced by $\ell$ to be

$$
\operatorname{lcr}_{i j}=\frac{\ell_{i l} \ell_{j k}}{\ell_{l j} \ell_{k i}} .
$$

This definition implicitly assumes that an orientation of the triangulated surface has been chosen. The other choice of orientation leads to reciprocal values for the lengthcross-ratios. (For nonorientable surfaces, the length-cross-ratios are well defined on the interior edges of the oriented double cover.) 
If the quadrilateral $i l j k$ is embedded in $\mathbb{C}$, then the length-cross-ratio $\operatorname{lcr}_{i j}$ is just the absolute value of the complex cross ratio of the vertex positions $z_{i}, z_{l}, z_{j}, z_{k}$,

$$
\operatorname{cr}\left(z_{1}, z_{2}, z_{3}, z_{4}\right)=\frac{\left(z_{1}-z_{2}\right)\left(z_{3}-z_{4}\right)}{\left(z_{2}-z_{3}\right)\left(z_{4}-z_{1}\right)} .
$$

Discretely conformally equivalent metrics $\ell, \tilde{\ell}$ induce the same length-cross-ratios, because the scale factors $e^{u / 2}$ cancel. By the reasoning above, the converse is also true.

Proposition 2.3.2 Two euclidean triangulations $(\mathrm{T}, \ell)$ and $(\mathrm{T}, \tilde{\ell})$ are discretely conformally equivalent if and only if for each interior edge $i j \in E_{\mathrm{T}}$, the induced lengthcross-ratios are equal: $\operatorname{lcr}_{i j}=\widetilde{\operatorname{lcr}}_{i j}$.

\subsection{The product of length-cross-ratios around a vertex}

Let us denote the sets of interior edges and interior vertices by $E_{\text {int }}$ and $V_{\text {int }}$, respectively. Which functions $E_{\text {int }} \rightarrow \mathbb{R}_{>0}$ can arise as length-cross-ratios? A necessary condition is that the product of length-cross-ratios on the edges around an interior vertex is 1 , because all lengths $\ell$ cancel:

$$
\text { for all } i \in V_{\mathrm{int}}, \quad \prod_{j: i j \in E} \operatorname{lcr}_{i j}=1 .
$$

If we ignore the triangle inequalities, this condition is also sufficient:

Proposition 2.4.1 Let lcr: $E_{\text {int }} \rightarrow \mathbb{R}_{>0}$ be any positive function on the set of interior edges. There exists a positive function $\ell: E \rightarrow \mathbb{R}_{>0}$ on the set of edges satisfying (2-4) for every interior edge $i j$ if and only if condition (2-5) holds.

Proof It remains to show that if $1 c r \in\left(\mathbb{R}_{>0}\right) E_{\text {int }}$ satisfies condition (2-5), then the system of equations (2-4) has a solution. In fact, we will explicitly construct such a solution. To this end, we introduce auxiliary parameters $c$, which are defined on the set of angles $A$ of the triangulation: given $\ell \in\left(\mathbb{R}_{>0}\right)^{E}$, define $c \in\left(\mathbb{R}_{>0}\right)^{A}$ by

$$
c_{j k}^{i}=\frac{\ell_{j k}}{\ell_{i j} \ell_{k i}}
$$

see Figure 4.

In terms of these parameters, the length-cross-ratios induced by $\ell$ are

$$
\operatorname{lcr}_{i j}=\frac{c_{j k}^{i}}{c_{l j}^{i}},
$$




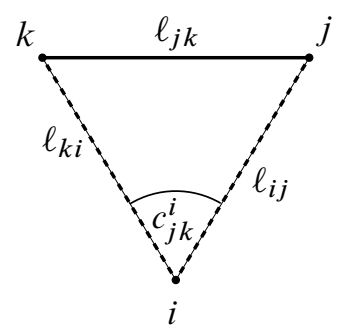

Figure 4: The parameters $c_{j k}^{i}$, defined on the set of triangle angles $A$

where $l, j, k$ occur in the link of $i$ in this cyclic order, as in Figure 3. (For a geometric interpretation of the parameters $c_{j k}^{i}$ in terms of hyperbolic geometry, see Section 5.2.)

Now suppose $\operatorname{lcr} \in\left(\mathbb{R}_{>0}\right){ }^{E_{\text {int }}}$ satisfies condition (2-5). Then it is easy to find a solution $c \in\left(\mathbb{R}_{>0}\right)^{A}$ of equations (2-7), because each equation involves only two values of $c$ on consecutive angles at the same vertex. So one can freely choose one $c$-value per vertex and successively calculate the values on neighboring angles around the same vertex by multiplying (or dividing) with the values of lcr on the edges in between.

Next, solve equations (2-6) for $\ell$, where $c$ is the solution to equations (2-7) just constructed. This is also easy: the length of an edge $i j$ is determined by the values of $c$ on the two adjacent angles on either side:

$$
\ell_{i j}=\left(c_{j k}^{i} c_{k i}^{j}\right)^{-1 / 2}
$$

(Check that the two $c$-values on the other side give the same value.) Thus we have constructed a function $\ell \in\left(\mathbb{R}_{>0}\right)^{E}$ satisfying equations (2-4) for the given function $\operatorname{lcr} \in\left(\mathbb{R}_{>0}\right)^{E_{\text {int }}}$.

\subsection{Möbius invariance of discrete conformal structures}

The group of Möbius transformations of $\widehat{\mathbb{R}}^{n}=\mathbb{R}^{n} \cup\{\infty\}$ is the group generated by inversions in spheres. (Planes are considered spheres through $\infty$.) The group of Möbius transformations is also generated by the similarity transformations (which fix $\infty$ ), and inversion in the unit sphere. Möbius transformations are conformal, and a famous theorem of Liouville says that for $n>2$, any conformal map of a domain $U \subset \mathbb{R}^{n}$ is the restriction of a Möbius transformation.

Let $\mathrm{T}$ be a triangulation and let $\|\cdot\|$ denote the euclidean norm on $\mathbb{R}^{n}, n \geq 2$. Suppose $v: V_{\mathrm{T}} \rightarrow \mathbb{R}^{n}$ maps the vertices of each triangle to three affinely independent points. Then $v$ induces a discrete metric $\ell_{i j}=\left\|v_{i}-v_{j}\right\|$. Two maps $v, \tilde{v}: V \rightarrow \mathbb{R}^{n} \subset \widehat{\mathbb{R}}^{n}$ are related by a Möbius transformation if there is a Möbius transformation $T$ such that $\tilde{v}=T \circ v$. 
Proposition 2.5.1 If two maps $V_{\mathrm{T}} \rightarrow \mathbb{R}^{n}$ are related by a Möbius transformation, then the induced discrete metrics are discretely conformally equivalent.

Proof The claim is obvious if the relating Möbius transformation is a similarity transformation. For inversion in the unit sphere, $x \mapsto \frac{1}{\|x\|^{2}} x$, it follows from the identity

$$
\left\|\frac{1}{\|p\|^{2}} p-\frac{1}{\|q\|^{2}} q\right\|=\frac{1}{\|p\|\|q\|}\|p-q\| \text {. }
$$

Remark 2.5.2 For $n=2$ there is an obvious alternative argument involving the complex cross ratio. One can extend this argument to $n>2$. The only difficulty is to define a complex cross-ratio for four points in $\mathbb{R}^{n}$ if $n>2$, such that it is invariant under Möbius transformations. Such a cross-ratio can be defined up to complex conjugation by identifying a 2-sphere through the four points conformally with the extended complex plane $\widehat{\mathbb{C}}$. This involves several choices: a choice of 2 -sphere if the four points are cocircular, a choice of orientation of the 2 -sphere, and choice of orientation-preserving conformal map to $\widehat{\mathbb{C}}$. Only the choice of orientation makes a difference, the two choices leading to conjugate values for the cross-ratio. The length-cross-ratio is the absolute value of this complex cross-ratio, so the ambiguity with respect to complex conjugation does not matter.

\subsection{Discrete conformal maps}
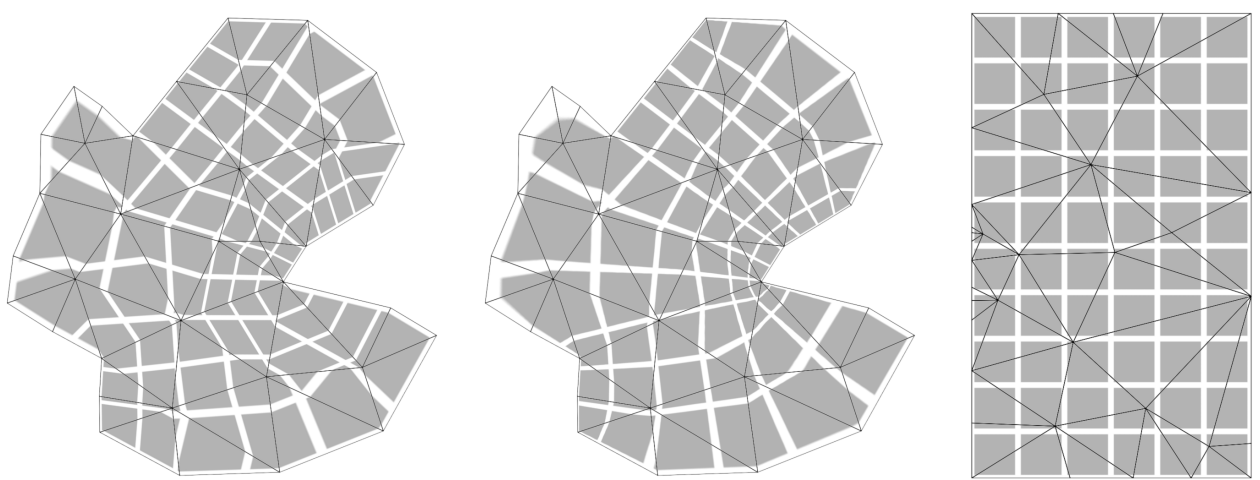

Figure 5: A coarsely triangulated domain in the plane (middle) is mapped to a rectangle (right) by a discrete conformal map (see Definition 2.6.1). Instead of using circumcircle-preserving piecewise projective interpolation, one can also interpolate linearly in each triangle. The result (left) looks noticeably "less smooth". 
So far we have only talked about discrete conformal equivalence. This section deals with the matching notion of discrete conformal maps (see Figure 5).

For any two euclidean triangles (with labeled vertices to indicate which vertices should be mapped to which), there is a unique projective map that maps one triangle onto the other and the circumcircle of one onto the circumcircle of the other (see Lemma 2.6.3 below). Let us call this map the circumcircle-preserving projective map between the two triangles.

Definition 2.6.1 A discrete conformal map from one euclidean triangulation $(\mathrm{T}, \ell)$ to a combinatorially equivalent euclidean triangulation $(\mathrm{T}, \tilde{\ell})$ is a homeomorphism whose restriction to every triangle is the circumcircle-preserving projective map onto the corresponding image triangle.

Consider two combinatorially equivalent euclidean triangulations $(\mathrm{T}, \ell)$ and $(\mathrm{T}, \tilde{\ell})$. For each individual triangle of $(T, \ell)$, there is a circumcircle-preserving projective map to the corresponding triangle of $(\mathrm{T}, \widetilde{\ell})$. But these maps do in general not fit together continuously across edges. However, they do fit together, forming a discrete conformal map, precisely if the euclidean triangulations are discretely conformally equivalent:

Theorem 2.6.2 The following two statements are equivalent.

(i) $(\mathrm{T}, \ell)$ and $(\mathrm{T}, \tilde{\ell})$ are discretely conformally equivalent.

(ii) There exists a discrete conformal map $(\mathrm{T}, \ell) \rightarrow(\mathrm{T}, \tilde{\ell})$.

The rest of this section is concerned with the proof of Theorem 2.6.2. It follows easily from Lemma 2.6.3 below, which provides an analytic description of the circumcirclepreserving projective map between two individual triangles.

Consider two triangles $\Delta$ and $\widetilde{\Delta}$ in the euclidean plane, and let $\left(x_{i}, y_{i}\right)$ and $\left(\tilde{x}_{i}, \tilde{y}_{i}\right)$, $i \in\{1,2,3\}$, be the coordinates of their vertices in a Cartesian coordinate system. Let $\ell_{i j}$ and $\tilde{\ell}_{i j}$ be the side lengths,

$$
\ell_{i j}^{2}=\left(x_{i}-x_{j}\right)^{2}+\left(y_{i}-y_{j}\right)^{2},
$$

and similarly for $\tilde{\ell}_{i j}$. Consider the euclidean plane as embedded in the projective plane $\mathbb{R} \mathrm{P}^{2}$ and let $v_{i}=\left(x_{i}, y_{i}, 1\right)$ and $\tilde{v}_{i}=\left(\tilde{x}_{i}, \tilde{y}_{i}, 1\right)$ be the homogeneous coordinate vectors of the vertices, normalized so that the last coordinate is 1 . Then the projective maps $f: \mathbb{R} \mathrm{P}^{2} \rightarrow \mathbb{R}^{2}$ that map $\Delta$ to $\tilde{\Delta}$ correspond via $f([v])=[F(v)]$ to the linear maps $F: \mathbb{R}^{3} \rightarrow \mathbb{R}^{3}$ of homogeneous coordinates that satisfy

$$
F\left(v_{i}\right)=\mu_{i} \widetilde{v}_{i}
$$

for some "weights" $\mu_{i} \in \mathbb{R} \backslash\{0\}$. 
Lemma 2.6.3 The projective map $f:[v] \mapsto[F(v)]$ maps the circumcircle of $\Delta$ to the circumcircle of $\widetilde{\Delta}$ if and only if

$$
\left(\mu_{1}, \mu_{2}, \mu_{3}\right)=\mu\left(e^{-u_{1}}, e^{-u_{2}}, e^{-u_{3}}\right),
$$

where $u_{1}, u_{2}, u_{3}$ are the logarithmic scale factors satisfying the three equations (2-1) for a single triangle and $\mu \in \mathbb{R} \backslash\{0\}$ is an arbitrary factor.

Proof of Lemma 2.6.3 The circumcircle of $\Delta$ is

$$
\left\{[v] \in \mathbb{R} \mathrm{P}^{2} \mid q(v)=0\right\},
$$

where $q$ is the quadratic form

$$
q(x, y, z)=x^{2}+y^{2}+2 a x z+2 b y z+c z^{2}
$$

with $a, b, c \in \mathbb{R}$ uniquely determined by the condition that

$$
q\left(v_{1}\right)=q\left(v_{2}\right)=q\left(v_{3}\right)=0 .
$$

In the same fashion, let the quadratic form describing the circumcircle of $\widetilde{\Delta}$ be

$$
\widetilde{q}(x, y, z)=x^{2}+y^{2}+2 \widetilde{a} x z+2 \tilde{b} y z+\widetilde{c} z^{2}
$$

so that

$$
\widetilde{q}\left(\widetilde{v}_{1}\right)=\widetilde{q}\left(\widetilde{v}_{2}\right)=\widetilde{q}\left(\widetilde{v}_{3}\right)=0 .
$$

We will also denote by $q$ and $\tilde{q}$ the corresponding symmetric bilinear forms:

$$
q(v)=q(v, v) \quad \text { and } \quad \tilde{q}(v)=\widetilde{q}(v, v) .
$$

The projective map $f$ maps circumcircle to circumcircle if and only if $q$ and the pullback $F^{*} \tilde{q}$ are linearly dependent. That is, if and only if

$$
\mu^{2} q(v, w)=\widetilde{q}(F(v), F(w))
$$

for all $v, w \in \mathbb{R}^{3}$ for some $\mu \in \mathbb{R}$. Since $v_{1}, v_{2}, v_{3}$ is a basis of $\mathbb{R}^{3}$ and because of equations (2-10) and (2-11), this is the case if and only if

$$
\mu^{2} q\left(v_{i}, v_{j}\right)=\mu_{i} \mu_{j} \widetilde{q}\left(\widetilde{v}_{i}, \widetilde{v}_{j}\right)
$$

for $i, j \in\{1,2,3\}, i \neq j$. Now note that

$$
\ell_{i j}^{2}=q\left(v_{i}-v_{j}, v_{i}-v_{j}\right)=-2 q\left(v_{i}, v_{j}\right),
$$

and similarly $\tilde{\ell}_{i j}^{2}=-2 \widetilde{q}\left(\widetilde{v}_{i}, \widetilde{v}_{j}\right)$. So condition (2-12) is equivalent to

$$
\mu^{2} \ell_{i j}^{2}=\mu_{i} \mu_{j} \tilde{\ell}_{i j}^{2}
$$


Solving equations (2-1) for $u_{i}$ to obtain (2-9) completes the proof of Lemma 2.6.3.

To prove Theorem 2.6.2, consider two euclidean triangulations $(\mathrm{T}, \ell)$ and $(T, \tilde{\ell})$, and a pair of adjacent triangles $i j k$ and $j i l$ of $T$. Embed the corresponding euclidean triangles of $(\mathrm{T}, \ell)$ simultaneously isometrically in the euclidean plane, and do the same for the corresponding two euclidean triangles of $(T, \tilde{\ell})$. Lemma 2.6.3 tells us what the circumcircle-preserving projective maps are, and we might as well choose $\mu=1$ in both cases. These two maps fit together continuously along edge $i j$ if and only if the values of $\mu_{i}=e^{-u_{i}}$ and $\mu_{j}=e^{-u_{j}}$ from one triangle are proportional to those of the other triangle. Since the value of $e^{\left(u_{i}+u_{j}\right) / 2}=\tilde{\ell}_{i j} / \ell_{i j}$ is the same for both triangles, this is the case if and only if the values of $\mu_{i}$ and $\mu_{j}$, hence also those of $u_{i}$ and $u_{j}$, coincide for both triangles. This holds for all interior edges if and only if $(\mathrm{T}, \ell)$ and $(T, \tilde{\ell})$ are discretely conformally equivalent. This completes the proof of Theorem 2.6.2.

\section{Discrete conformal mapping problems}

\subsection{Prescribing angle sums at vertices}

Consider the following type of discrete conformal mapping problem, which is a discrete version of the problem considered by Troyanov [44]:

Problem 3.1.1 (Prescribed angle sums) Given

- a surface triangulation $T$,

- a discrete conformal class $\mathcal{C}$ of discrete metrics on $\mathrm{T}$,

- a desired angle sum $\Theta_{i}$ for each vertex $i \in V_{\mathrm{T}}$,

find a discrete metric $\tilde{\ell}$ in the conformal class $\mathcal{C}$ such that the euclidean triangulation $(\mathrm{T}, \tilde{\ell})$ has angle sum $\Theta_{i}$ around each vertex $i \in V_{\mathrm{T}}$.

If, in particular, the given desired angle sum $\Theta_{i}$ equals $2 \pi$ for every interior vertex $i$, then Problem 3.1.1 asks for a flat euclidean triangulation in the given conformal class which has prescribed angles at the boundary. A flat and simply connected euclidean triangulation can be developed in the plane by laying out one triangle after the other. Thus, Problem 3.1.1 comprises as a special case the following problem.

Problem 3.1.2 (Planar triangulation with prescribed boundary angles) Given

- a euclidean triangulation $(T, \ell)$ that is topologically a disc

- a desired angle sum $\Theta_{i}$ for each boundary vertex $i$, 
find a discretely conformally equivalent planar triangulation $(\mathrm{T}, \tilde{\ell})$ with the given angle sums at the boundary. (The triangulated planar region may overlap with itself.)

We also consider a more general type of problem than Problem 3.1.1. Suppose the discrete conformal class $\mathcal{C}$ is given in the form of a representative metric $\ell \in\left(\mathbb{R}_{>0}\right)^{E}$. For some vertices $i$ we may prescribe the (logarithmic) scale factor $u_{i}$ instead of the angle sum $\Theta_{i}$ :

Problem 3.1.3 (Prescribed angles sums and fixed scale factors) Given

- a triangulation $\mathrm{T}$,

- a function $\ell \in\left(\mathbb{R}_{>0}\right)^{E}$ representing a conformal class,

- a partition $V=V_{0} \dot{U} V_{1}$ of the vertex set,

- a prescribed logarithmic scale factor $u_{i} \in \mathbb{R}$ for each vertex $i \in V_{0}$

- a prescribed angle sum $\Theta_{i}$ for each vertex $i \in V_{1}$,

find logarithmic scale factors $u_{i} \in \mathbb{R}$ for the remaining vertices $i \in V_{1}$ so that $\tilde{\ell}$ determined by equations (2-1) is a discrete metric and $(T, \tilde{\ell})$ has the prescribed angle sum $\Theta_{i}$ around each vertex $i \in V_{1}$.

For $V_{0}=\varnothing, V_{1}=V$, this is just Problem 3.1.1. (If the conformal class $\mathcal{C}$ is given in the form of length-cross-ratios $\operatorname{lcr} \in\left(\mathbb{R}_{>0}\right) E_{\text {int }}$, one can obtain a representative $\ell \in\left(\mathbb{R}_{>0}\right)^{E}$ using the method described in the constructive proof of Proposition 2.4.1.)

Note that any instance of Problem 3.1.3 can be reduced to the special case where $u_{i}=0$ is prescribed for $i \in V_{0}$ : simply apply first a discrete conformal change of metric (2-1) with the arbitrary prescribed $u_{i}$ for $i \in V_{0}$.

Analytically, Problem 3.1.3 amounts to solving a system of nonlinear equations. For the unknown logarithmic scale factors $u_{i}\left(i \in V_{1}\right)$, one has to solve the system of angle-sum equations

$$
\sum_{j k: i j k \in T} \tilde{\alpha}_{j k}^{i}=\Theta_{i}
$$

with one equation for each vertex $i \in V_{1}$. Here, $\widetilde{\alpha}_{j k}^{i}$ is the angle at $i$ in triangle $i j k$ of $(\mathrm{T}, \tilde{\ell})$. The angles $\tilde{\alpha}$ are nonlinear functions of the new lengths $\tilde{\ell}$. They can be obtained by invoking, for example, the cosine rule or the half-angle formula

$$
\tan \left(\frac{\alpha_{j k}^{i}}{2}\right)=\sqrt{\frac{\left(-\ell_{i j}+\ell_{j k}+\ell_{k i}\right)\left(\ell_{i j}+\ell_{j k}-\ell_{k i}\right)}{\left(\ell_{i j}-\ell_{j k}+\ell_{k i}\right)\left(\ell_{i j}+\ell_{j k}+\ell_{k i}\right)}} .
$$

(Tilde marks over $\alpha$ and $\ell$ have been omitted in this equation to avoid visual clutter.) 
Theorem 3.1.4 If Problem 3.1.3 has a solution, then the solution is unique if $V_{0} \neq \varnothing$ (ie at least one scale factor is fixed) and unique up to scale if $V_{0}=\varnothing$. The solution can be found by minimizing a convex function.

Proof This follows from Propositions 4.1.3, 4.1.4 and 4.1.5.

Corollary 3.1.5 If a solution to Problem 3.1.1 (or 3.1.2) exists, it is unique up to scale, and it can be found by minimizing a convex function.

Remark 3.1.6 An important special case of Problem 3.1.3 is the following: prescribe the angle sum $\Theta_{i}=2 \pi$ for interior vertices, and $u_{i}$ on the boundary. This is analogous to the following boundary value problem of the smooth theory: given a smooth $2-$ manifold with boundary $M$ equipped with a Riemannian metric $g$, find a conformally equivalent flat Riemannian metric $e^{2 u} g$ with prescribed $\left.u\right|_{\partial M}$. Suppose we measure the relative distortion of a conformally equivalent Riemannian metric by the Dirichlet energy of $u, D(u)=\frac{1}{2} \int_{M} d u \wedge * d u$. Then the conformally equivalent flat Riemannian metrics with least distortion are those with $\left.u\right|_{\partial M}=$ const. Thus, up to scale there is a unique least distortion solution obtained by setting $\left.u\right|_{\partial M}=0$ [40, Appendix E].

\subsection{Mapping to the sphere}

If one can solve Problem 3.1.3, one can also find discrete conformal maps from euclidean triangulations that are topological spheres to polyhedra with vertices on the unit sphere, and from euclidean triangulations that are topological disks to planar triangulations with boundary vertices on the unit circle (Section 3.3).

Suppose $(T, \ell)$ is a euclidean triangulation that is topologically a sphere. To map it to a polyhedron with vertices on the unit sphere, proceed as follows.

(1) Choose a vertex $k$ and apply a discrete conformal change of metric (2-1) so that afterwards all edges incident with $k$ have the same length, say $\tilde{\ell}_{k i}=1$ for all neighbors $i$ of $k$. For example, let $e^{u_{i} / 2}=\ell_{k i}^{-1}$ if $i$ is a neighbor of $k$ and 1 otherwise.

(2) Let $T^{\prime}$ be $T$ minus the open star of $k$. This is topologically a closed disk.

(3) Solve Problem 3.1.3 for $T^{\prime}$ with prescribed $\Theta_{i}=2 \pi$ for interior vertices $i$ and prescribed $u_{i}=0$ for boundary vertices. (Suppose a solution exists.) The result is a planar triangulation.

(4) Map the vertices of this planar triangulation to the unit sphere by stereographic projection. Add another vertex (the image of the removed vertex $k$ ) on the sphere at the center of the stereographic projection. Build a geometric simplicial complex using these vertices and the combinatorics of $\mathrm{T}$. 
Proposition 3.2.1 The result of this procedure is a polyhedron with vertices on the sphere that is discretely conformally equivalent to $(\mathrm{T}, \ell)$. (It may not be convex. It is also possible that the planar triangulation obtained in step three overlaps with itself. In this case the star of $k$ in the image polyhedron is not embedded.)

Proof After step (1), the length-cross-ratio for an edge $k i$ incident with $k$ is the quotient of the lengths of two consecutive edges $m i, i j$ in the boundary of $T^{\prime}$. This is not changed in step (3) because $u=0$ on the boundary. Further, the length-cross-ratio for an edge $i j$ opposite $k$ as in Figure 3 is then the quotient $\ell_{i l} / \ell_{l j}$. This is also not changed in step (3) because $u=0$ on the boundary. Now imagine that before step (4) you reinsert $k$ at $\infty$ in the plane, which you identify with the (extended) complex plane. Then the absolute values of the complex cross-ratios for all edges are the same as in $(T, \ell)$.

Remark 3.2.2 The method presented here is a variation of a method described [40]. The old version requires an input triangulation that is immersed in some $\mathbb{R}^{n}$ with straight edges.

\subsection{Mapping to the disk}

Suppose $(T, \ell)$ is a euclidean triangulation that is topologically a closed disk. To map it to a triangulated circular polygon, proceed as follows (see Figure 6):

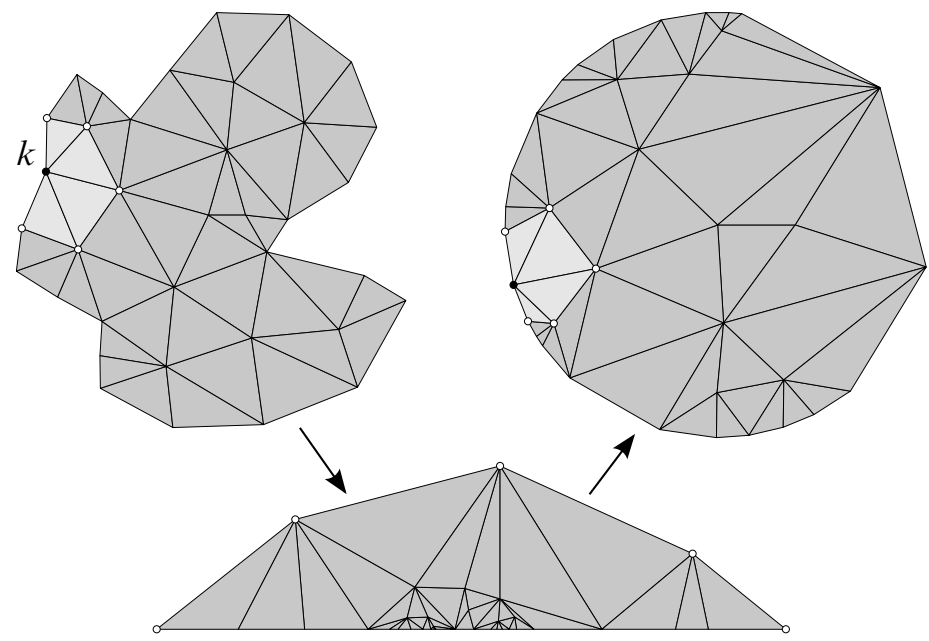

Figure 6: Mapping to the disk 
(1) Choose a boundary vertex $k$ and apply a discrete conformal change of metric (2-1) so that afterwards all edges incident with $k$ have the same length, say $\tilde{\ell}_{k i}=1$ for all neighbors $i$ of $k$. For example, let $e^{u_{i} / 2}=\ell_{k i}^{-1}$ if $i$ is a neighbor of $k$ and 1 otherwise.

(2) Let $T^{\prime}$ be $T$ minus the open star of $k$. Suppose this is topologically still a closed disk.

(3) Solve Problem 3.1.3 for $T^{\prime}$, with prescribed $\Theta_{i}=2 \pi$ for interior vertices of $T^{\prime}$, $\Theta_{i}=\pi$ for boundary vertices of $T^{\prime}$ that are not neighbors of $k$ in $\mathrm{T}$, and prescribed $u_{i}=0$ for the neighbors of $k$ in T. (Suppose a solution exists.) The result is a planar triangulation. All boundary edges except the neighbors of $k$ in $T$ are contained in one straight line.

(4) Apply a Möbius transformation to the vertices that maps this straight line to a circle and the other vertices inside this circle. Reinsert $k$ at the image point of $\infty$ under this Möbius transformation.

Proposition 3.3.1 The result of this procedure is a planar triangulation that is discretely conformally equivalent to $(\mathrm{T}, \ell)$ and has a boundary polygon that is inscribed in a circle.

We omit the proof because no new ideas are needed.

Remark 3.3.2 Note that for Problem 3.1.3 in step 3 to be solvable, the triangulation $T$ should not have any ears (ie triangles on the boundary that are attached by one edge only). Prescribing a total angle of $\pi$ at boundary vertices forces such triangles to degenerate.

\section{Two variational principles}

\subsection{The first variational principle}

The system of nonlinear equations described in the previous section turns out to be variational. Solutions of the conformal mapping problems correspond to the critical points of the function $E_{\mathrm{T}, \Theta, \lambda}$ defined as follows. The precise statement of this first variational principle is Proposition 4.1.3.

Let $\mathrm{T}$ be a surface triangulation, $\Theta \in \mathbb{R}^{V}$ and $\lambda \in \mathbb{R}^{E}$. For now (we will later extend the domain of definition to $\mathbb{R}^{V}$ ) define the real-valued function $E_{\mathrm{T}, \Theta, \lambda}(u)$ on the open 
subset of $\mathbb{R}^{V}$ containing all $u$ such that $\tilde{\ell}$ determined by (2-1) is a discrete metric (that is, satisfies the triangle inequalities):

$$
\begin{aligned}
E_{\mathrm{T}, \Theta, \lambda}(u)=\sum_{i j k \in T}\left(\widetilde{\alpha}_{i j}^{k} \tilde{\lambda}_{i j}+\right. & \tilde{\alpha}_{j k}^{i} \tilde{\lambda}_{j k}+\tilde{\alpha}_{k i}^{j} \tilde{\lambda}_{k i}+2 \pi\left(\tilde{\alpha}_{i j}^{k}\right)+2 \pi\left(\widetilde{\alpha}_{j k}^{i}\right) \\
& \left.+2 \pi\left(\tilde{\alpha}_{k i}^{j}\right)-\frac{\pi}{2}\left(\tilde{\lambda}_{i j}+\tilde{\lambda}_{j k}+\tilde{\lambda}_{k i}\right)\right)+\sum_{i \in V} \Theta_{i} u_{i} .
\end{aligned}
$$

The first sum is taken over all triangles, $\widetilde{\alpha}_{j k}^{i}$ denotes the angle at vertex $i$ in triangle $i j k$ with side lengths $\tilde{\ell}=e^{\tilde{\lambda} / 2}$,

$$
\tilde{\lambda}_{i j}=\lambda_{i j}+u_{i}+u_{j}
$$

and $Л(x)$ is Milnor's Lobachevsky function,

$$
\pi(x)=-\int_{0}^{x} \log |2 \sin (t)| d t .
$$

(Figure 7 shows a graph of this function.) The second sum is taken over all vertices. It is linear in $u$.

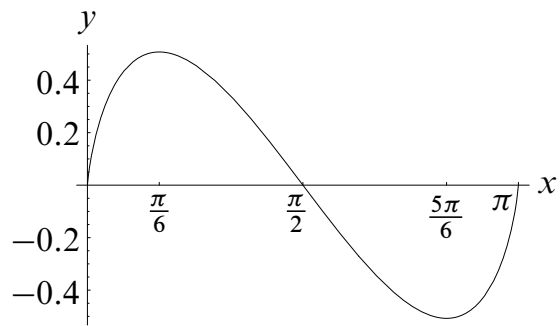

Figure 7: Graph of Milnor's Lobachevsky function, $y=Л(x)$ : it's $\pi-$ periodic, odd and smooth except at $x \in \pi \mathbb{Z}$, where its tangents are vertical.

Remark 4.1.1 The notation $Л(x)$, using a letter from the Cyrillic alphabet, and the name "Lobachevsky function" are due to Milnor $[27 ; 28]$. Lobachevsky used a slightly different function which is also known as the Lobachevsky function and often denoted $L(x)$. To distinguish these two functions, we call $Л(x)$ Milnor's Lobachevsky function. It is almost the same as Clausen's integral (see Lewin [23]), $\mathrm{Cl}_{2}(x)=\frac{1}{2} \pi(2 x)$.

Proposition 4.1.2 (First derivative) The partial derivative of $E_{\mathrm{T}, \Theta, \lambda}$ with respect to $u_{i}$ is

$$
\frac{\partial}{\partial u_{i}} E_{\mathrm{T}, \Theta, \lambda}=\Theta_{i}-\sum_{j k: i j k \in T} \widetilde{\alpha}_{j k}^{i},
$$

where the sum is taken over all angles around vertex $i$. 
Proof This follows from Equation (4-6) and Proposition 4.2.1.

Proposition 4.1.3 (First variational principle) Let $\mathcal{C}$ be a discrete conformal class with representative metric $\ell=e^{\lambda / 2}$, and let $\tilde{\ell}=e^{\tilde{\lambda} / 2}$ where $\tilde{\lambda}$ is the function of $u$ defined by equations (2-3). Then:

- $\tilde{\ell}$ solves Problem 3.1.1 if and only if $u$ is a critical point of $E_{\mathrm{T}, \Theta, \lambda}$.

- $\tilde{\ell}$ solves Problem 3.1.3 if and only if $u$ is a critical point of $E_{\mathrm{T}, \Theta, \lambda}$ with fixed $u_{i}$ for $i \in V_{0}$. (In this case, the values of $\Theta$ for $i \in V_{0}$ are irrelevant.)

Proof This follows immediately from Proposition 4.1.2 because $\frac{\partial}{\partial u_{i}} E_{\mathrm{T}, \Theta, \lambda}=0$ is equivalent to the angle sum condition (3-1).

Proposition 4.1.4 (Local convexity) The function $E_{\mathrm{T}, \Theta, \lambda}$ is locally convex, that is, its second derivative $\sum \partial^{2} E_{\mathrm{T}, \Theta, \lambda} / \partial u_{i} \partial u_{j} d u_{i} d u_{j}$ is a positive semidefinite quadratic form. The kernel is 1-dimensional and consists of the constants in $\mathbb{R}^{V}$.

Proof This follows from Equation (4-6) and Proposition 4.2.4.

Proposition 4.1.5 (Extension) The function $E_{\mathrm{T}, \Theta, \lambda}$ can be extended to a convex continuously differentiable function on $\mathbb{R}^{V}$.

Proof This follows from Equation (4-6) and Proposition 4.2.5.

In fact, one has an explicit formula for the second derivative of $E_{\mathrm{T}, \Theta, \lambda}$. This is helpful from the practical point of view, because it allows one to use more powerful algorithms to minimize $E_{\mathrm{T}, \Theta, \lambda}$ and thus solve the discrete conformal mapping problems. It is also interesting from the theoretical point of view, because the second derivative of $E_{\mathrm{T}, \Theta, \lambda}$ at $u$ is the well-known finite-element approximation of the Dirichlet energy (the cotan-formula) for a triangulation with edge lengths $\tilde{\ell}$; see Duffin [10] and the second author and Polthier [33]:

Proposition 4.1.6 (Second derivative) The second derivative of $E_{\mathrm{T}, \Theta, \lambda}$ at $u$ is

$$
\sum_{i, j \in V} \frac{\partial^{2} E_{\mathrm{T}, \Theta, \lambda}}{\partial u_{i} \partial u_{j}} d u_{i} d u_{j}=\frac{1}{2} \sum_{i j \in E} w_{i j}(u)\left(d u_{i}-d u_{j}\right)^{2},
$$

where $w_{i j}(u)=\frac{1}{2}\left(\cot \widetilde{\alpha}_{i j}^{k}+\cot \widetilde{\alpha}_{i j}^{l}\right)$ if $i j$ is an interior edge with opposite vertices $k$ and $l$ and $w_{i j}(u)=\frac{1}{2} \cot \widetilde{\alpha}_{i j}^{k}$ if $i j$ is a boundary edge with opposite vertex $k$. This assumes all triangle inequalities are satisfied. If triangle inequalities are violated, the cotangent terms for the corresponding triangles have to be replaced with 0 .

Proof This follows from Equation (4-6) and Proposition 4.2.3. 


\subsection{A peculiar triangle function}

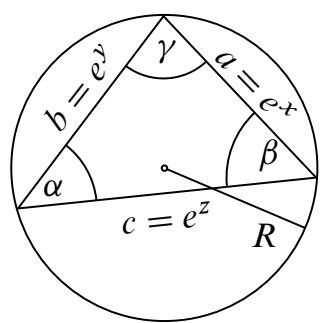

Figure 8: Triangle with sides $a=e^{x}, b=e^{y}, c=e^{z}$ and angles $\alpha, \beta, \gamma$ : the radius of the circumcircle is $R=\frac{a}{2 \sin \alpha}=\frac{b}{2 \sin \beta}=\frac{c}{2 \sin \gamma}$.

Consider the function

$$
f(x, y, z)=\alpha x+\beta y+\gamma z+\pi(\alpha)+\pi(\beta)+\pi(\gamma),
$$

where $\alpha, \beta$ and $\gamma$ are the angles in a euclidean triangle with sides $a=e^{x}, b=e^{y}$ and $c=e^{z}$ as shown in Figure 8. Such a triangle exists if and only if the triangle inequalities are satisfied. So $f$ is (for now) only defined on the set

(4-4) $\mathcal{A}=\left\{(x, y, z) \in \mathbb{R}^{3} \mid-e^{x}+e^{y}+e^{z}>0, e^{x}-e^{y}+e^{z}>0, e^{x}+e^{y}-e^{z}>0\right\}$.

Note that the function $f(x, y, z)$ is affine in the $(1,1,1)$-direction:

$$
f(x+h, y+h, z+h)=f(x, y, z)+\pi h .
$$

This equation remains valid after the extension of $f$ to $\mathbb{R}^{3}$ described in Proposition 4.2.5. We will use it in Appendix A to prove Proposition A.1.1.

The function $f(x, y, z)$ is the fundamental building block of $E_{\mathrm{T}, \Theta, \lambda}(u)$ since

(4-6) $E_{\mathrm{T}, \Theta, \lambda}(u)=\sum_{i j k \in T}\left(2 f\left(\frac{\tilde{\lambda}_{i j}}{2}, \frac{\tilde{\lambda}_{j k}}{2}, \frac{\tilde{\lambda}_{k i}}{2}\right)-\frac{\pi}{2}\left(\tilde{\lambda}_{i j}+\tilde{\lambda}_{j k}+\tilde{\lambda}_{k i}\right)\right)+\sum_{i \in V} \Theta_{i} u_{i}$,

and Propositions 4.1.2, 4.1.4, 4.1.5 and 4.1.6 follow from corresponding statements regarding $f(x, y, z)$.

Proposition 4.2.1 (First derivative) The partial derivatives of $f$ are

$$
\frac{\partial f}{\partial x}=\alpha, \quad \frac{\partial f}{\partial y}=\beta, \quad \frac{\partial f}{\partial z}=\gamma .
$$


Proof Using $\pi^{\prime}(x)=-\log |2 \sin (x)|$ we obtain from (4-3) that

$$
\frac{\partial f}{\partial x}=\alpha+(x-\log (2 \sin \alpha)) \frac{\partial \alpha}{\partial x}+(y-\log (2 \sin \beta)) \frac{\partial \beta}{\partial x}+(z-\log (2 \sin \gamma)) \frac{\partial \gamma}{\partial x} .
$$

Since

$$
x-\log (2 \sin \alpha)=y-\log (2 \sin \beta)=z-\log (2 \sin \gamma)=\log R,
$$

where $R$ is the radius of the circumcircle, and since

$$
\frac{\partial \alpha}{\partial x}+\frac{\partial \beta}{\partial x}+\frac{\partial \gamma}{\partial x}=0
$$

(because $\alpha+\beta+\gamma=\pi$ ), this implies $\frac{\partial f}{\partial x}=\alpha$.

Remark 4.2.2 All closed one-forms of the form $\sum_{i=1}^{3} f\left(\alpha_{i}\right) d g\left(\ell_{i}\right)$, where $\ell_{1}, \ell_{2}, \ell_{3}$, and $\alpha_{1}, \alpha_{2}, \alpha_{3}$ are the sides and angles of a (euclidean, hyperbolic, or spherical) triangle, have been classified by Luo [25]; see also Dai, Gu and Luo [9]. In the euclidean case, they are the one-forms $w_{s}=\sum_{i=1}^{3}\left(\int^{\alpha_{i}} \sin ^{s} t d t\right) d \ell_{i} / \ell_{i}^{s+1}$. Thus, the function $f$ is the integral of $w_{0}$.

Proposition 4.2.3 (Second derivative) The second derivative of $f$ is

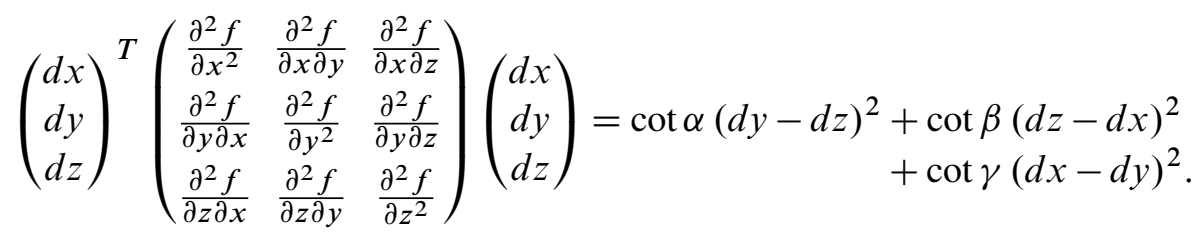

Proof By Proposition 4.2.1,

$$
\left(\begin{array}{c}
d x \\
d y \\
d z
\end{array}\right)^{T}\left(\begin{array}{ccc}
\frac{\partial^{2} f}{\partial x^{2}} & \frac{\partial^{2} f}{\partial x \partial y} & \frac{\partial^{2} f}{\partial x \partial z} \\
\frac{\partial^{2} f}{\partial y \partial x} & \frac{\partial^{2} f}{\partial y^{2}} & \frac{\partial^{2} f}{\partial y \partial z} \\
\frac{\partial^{2} f}{\partial z \partial x} & \frac{\partial^{2} f}{\partial z \partial y} & \frac{\partial^{2} f}{\partial z^{2}}
\end{array}\right)=\left(\begin{array}{lll}
d \alpha & d \beta & d \gamma
\end{array}\right)
$$

so the left-hand side of equation (4-7) equals

$$
d \alpha d x+d \beta d y+d \gamma d z
$$

We will show that

$$
d \alpha=\cot \gamma(d x-d y)+\cot \beta(d x-d z) .
$$

This and the analogous equations for $d \beta$ and $d \gamma$ imply $d \alpha d x+d \beta d y+d \gamma d z=\cot \alpha(d y-d z)^{2}+\cot \beta(d z-d x)^{2}+\cot \gamma(d x-d y)^{2}$, 
and hence Equation (4-7).

To derive Equation (4-8), differentiate the cosine rule

$$
2 b c \cos \alpha=b^{2}+c^{2}-a^{2}
$$

to get

$-2 b c \sin \alpha d \alpha+2 b c \cos \alpha(d y+d z)=2 b^{2} d y+2 c^{2} d z-2 a^{2} d x$.

Apply the cosine rule three more times to get

$$
\begin{aligned}
2 b c \sin \alpha d \alpha & =\left(b^{2}-c^{2}+a^{2}\right)(d x-d y)+\left(-b^{2}+c^{2}+a^{2}\right)(d x-d z) \\
& =2 a b \cos \gamma(d x-d y)+2 a c \cos \beta(d x-d z) .
\end{aligned}
$$

Divide through by $2 b c \sin \alpha$ and apply the sine rule to obtain Equation (4-8).

Proposition 4.2.4 (Local convexity) The function $f$ is locally convex, that is, the second derivative (4-7) is a positive semidefinite quadratic form. Its kernel is onedimensional and spanned by $(1,1,1) \in \mathbb{R}^{3}$.

Proof Writing $(d y-d z)$ as $((d y-d x)-(d x-d z))$ we obtain

$$
\begin{aligned}
\cot \alpha(d y-d z)^{2}+\cot \beta(d z-d x)^{2}+\cot \gamma(d x-d y)^{2} & \\
=(\cot \alpha+\cot \beta)(d x-d z)^{2}+(\cot \alpha+\cot \gamma)(d x-d y)^{2} & \\
& -2 \cot \alpha(d x-d y)(d x-d z) .
\end{aligned}
$$

Thus, in terms of $(d x-d z)$ and $(d x-d y)$, the matrix of this quadratic form is

$$
M=\left(\begin{array}{cc}
\cot \alpha+\cot \beta & -\cot \alpha \\
-\cot \alpha & \cot \alpha+\cot \gamma
\end{array}\right) \text {. }
$$

We proceed as in [34, Section 2]. Using $\alpha+\beta+\gamma=\pi$, we obtain

$$
M=\frac{1}{\sin \alpha \sin \beta \sin \gamma}\left(\begin{array}{cc}
\sin ^{2} \gamma & -\cos \alpha \sin \beta \sin \gamma \\
-\cos \alpha \sin \beta \sin \gamma & \sin ^{2} \beta
\end{array}\right)
$$

and $\operatorname{det} M=1$. Since $M_{11}>0$ and $\operatorname{det} M>0, M$ is positive definite. The claim about the second derivative of $f$ follows.

Proposition 4.2.5 (Extension) Extend the definition of $f$ from $\mathcal{A}$ to $\mathbb{R}^{3}$ as follows. Define $f(x, y, z)$ by Equation (4-3) for all $(x, y, z) \in \mathbb{R}^{3}$, where for $(x, y, z) \notin \mathcal{A}$ the angles $\alpha, \beta$ and $\gamma$ are defined to be $\pi$ for the angle opposite the side that is too long and 0 for the other two. The so extended function $f: \mathbb{R}^{3} \rightarrow \mathbb{R}$ is continuously differentiable and convex. 
Proof The so-defined functions $\alpha, \beta, \gamma$ are continuous on $\mathbb{R}^{3}$. This implies the continuity of $f$ and, together with Proposition 4.2.1, the continuity of its first derivative. Since $f$ is locally convex in $\mathcal{A}$ (Proposition 4.2.4) and linear outside, it is convex.

Figure 9 shows contour lines of the extended function $f(x, y, z)$ in the plane $z=0$ and its graph.
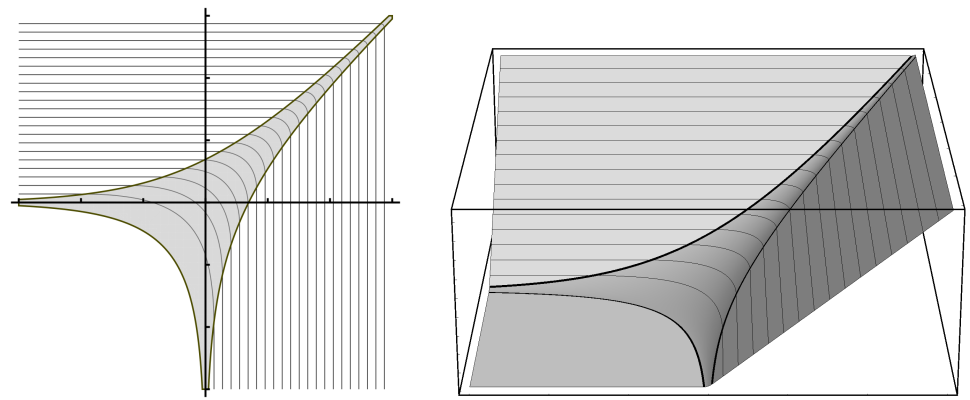

Figure 9: On the left, we have the contour plot of $(x, y) \mapsto f(x, y, 0)$ : the intersection of the domain $\mathcal{A}$ (see equation (4-4)) with the $x y$-plane is shaded. On the right, we have the graph of the same function, also showing contour lines.

We will need the following estimate in the proof of Proposition A.2.2.

Proposition 4.2.6 (Estimate) We have $f(x, y, z) \geq \pi \max \{x, y, z\}$.

Proof The inequality follows from Proposition 4.2.5 and the following two observations. First, the condition of convexity,

$$
f\left((1-t) p_{1}+t p_{2}\right) \leq(1-t) f\left(p_{1}\right)+t f\left(p_{2}\right) \quad \text { if } 0 \leq t \leq 1,
$$

is equivalent to

$$
f\left((1-t) p_{1}+t p_{2}\right) \geq(1-t) f\left(p_{1}\right)+t f\left(p_{2}\right) \quad \text { if } t \leq 0 \text { or } t \geq 1 .
$$

Second, for fixed $y$ and $z$, if $x$ is greater than some constant, then $f(x, y, z)=\pi x$. Together, they imply $f(x, y, z) \geq \pi x$. Equally, we have $f(x, y, z) \geq \pi y$ and $f(x, y, z) \geq \pi z$.

Remark 4.2.7 (Amoebas and Ronkin functions) In fact, $\mathcal{A}$ is an amoeba, the extended $f$ is a Ronkin function, and the convexity of $f$ that we have proved by elementary means follows also from a general theorem of Passare and Rullgård [31], 
which says that a Ronkin function is convex. Amoebas were introduced by Gelfand, Kapranov and Zelevinsky [15]. The amoeba $\mathcal{A}_{p}$ of a complex polynomial $p\left(z_{1}, \ldots, z_{n}\right)$ with $n$ indeterminates is defined as the domain in $\mathbb{R}^{n}$ that is the image of the set of zeros of $p$ under the map $\left(z_{1}, \ldots, z_{n}\right) \mapsto\left(\log \left|z_{1}\right|, \ldots, \log \left|z_{n}\right|\right)$. So the domain $\mathcal{A}$ defined by Equation (4-4) is the amoeba of the linear polynomial $z_{1}+z_{2}+z_{3}$. The Ronkin function of a polynomial $p$ is defined as the function $N_{p}: \mathbb{R}^{n} \rightarrow \mathbb{R}$,

$$
N_{p}\left(x_{1}, \ldots, x_{n}\right)=\frac{1}{(2 \pi i)^{n}} \int_{S^{1}\left(e^{x_{1}}\right) \times \cdots \times S^{1}\left(e^{x_{n}}\right)} \log \left|p\left(z_{1}, \ldots, z_{n}\right)\right| \frac{d z_{1}}{z_{1}} \wedge \cdots \wedge \frac{d z_{n}}{z_{n}},
$$

where $S^{1}(r)$ is the circle in $\mathbb{C}$ around 0 with radius $r$. As it turns out,

$$
f\left(x_{1}, x_{2}, x_{3}\right)=\pi N_{z_{1}+z_{2}+z_{3}}\left(x_{1}, x_{2}, x_{3}\right) .
$$

We will not spoil the reader's fun by presenting a proof here. The same Ronkin function also appears in the work of Kenyon, Okounkov and Sheffield on the dimer model (see $[19 ; 20])$ as the Legendre dual of a "surface tension" in a variational principle governing the limit shape of random surfaces. (See in particular Kenyon's survey article on dimers [18] and Mikhalkin's survey article on amoebas [26].) Whether or how this is related to the variational principles discussed in this paper is unclear.

\subsection{The second variational principle}

The second variational principle has angles as variables. It is based on the two elementary observations that, first, the sine theorem lets us express the length-cross-ratios in terms of angles,

$$
\operatorname{lcr}_{i j}=\frac{\sin \left(\alpha_{i l}^{j}\right) \sin \left(\alpha_{j k}^{i}\right)}{\sin \left(\alpha_{l j}^{i}\right) \sin \left(\alpha_{k i}^{j}\right)}
$$

(see Figure 10), and that, second, if we know the angles in a euclidean triangulation, then we can (again using the sine theorem) reconstruct the lengths up to a global scale factor.

For a triangulated surface $\mathrm{T}$ and $\lambda \in \mathbb{R}^{E}$, define

$$
\begin{aligned}
S_{\mathrm{T}, \lambda}: \mathbb{R}^{A} & \rightarrow \mathbb{R}, \\
S_{\mathrm{\top}, \lambda}(\alpha) & =\sum_{i j k \in T}\left(2 V\left(\alpha_{i j}^{k}, \alpha_{j k}^{i}, \alpha_{k i}^{j}\right)+\alpha_{i j}^{k} \lambda_{i j}+\alpha_{j k}^{i} \lambda_{j k}+\alpha_{k i}^{j} \lambda_{k i}\right),
\end{aligned}
$$

where

$$
V(\alpha, \beta, \gamma)=\pi(\alpha)+\pi(\beta)+\pi(\gamma)
$$




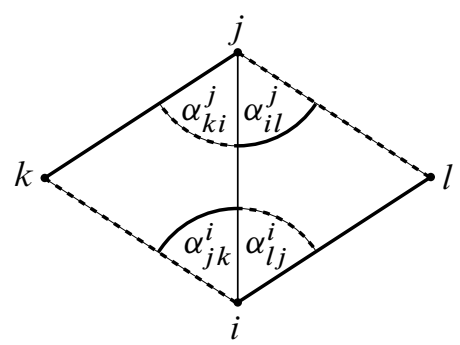

Figure 10: The sine theorem lets us express the length-cross-ratios (see Figure 3) in terms of angles.

Remark 4.3.1 The function $S_{\mathrm{T}, \lambda}$ is (up to an irrelevant additive constant) equal to Rivin's function $\mathcal{V}_{S}$ defined in [34, Section 7]. But the variational principles considered here (Propositions 4.3.3 and 4.3.4) are different. Rivin's variational principle has an additional constraint: only such variations are allowed that fix, for each edge, the sum of opposite angles.

Proposition 4.3.2 (Rivin [34]) The function $V$ is strictly concave on the domain

$$
\left\{(\alpha, \beta, \gamma) \in\left(\mathbb{R}_{>0}\right)^{3} \mid \alpha+\beta+\gamma=\pi\right\} .
$$

So $S_{\mathrm{T}, \lambda}$ is also strictly concave on the domain of positive angle assignments that sum to $\pi$ in each triangle.

Proposition 4.3.3 (Second variational principle I) Let $\mathcal{C}$ be a discrete conformal class on $\mathrm{T}$ with representative $\ell=e^{\lambda / 2} \in \mathbb{R}^{E}$, let $\Theta \in \mathbb{R}^{V}$, and define the subset $C_{\Theta} \subseteq \mathbb{R}^{A}$ by

$$
\begin{aligned}
& C_{\Theta}=\left\{\alpha \in \mathbb{R}^{A} \mid \alpha>0, \text { for all } i j k \in T: \alpha_{j k}^{i}+\alpha_{k i}^{j}+\alpha_{i j}^{k}=\pi,\right. \\
& \left.\qquad \text { for all } i \in V: \sum_{j k: i j k \in T} \alpha_{j k}^{i}=\Theta_{i}\right\}
\end{aligned}
$$

Then $\tilde{\alpha} \in C_{\Theta}$ is the angle function of a solution $\tilde{\ell}=e^{\tilde{\lambda} / 2}$ of Problem 3.1.1 if and only if $S_{\mathrm{T}, \lambda}(\widetilde{\alpha})$ is the maximum of the restriction $\left.S_{\mathrm{T}, \lambda}\right|_{C_{\Theta}}$.

Proof Consider the graph $\Gamma$ that is obtained by choosing one point in each triangle of $\mathrm{T}$ and connecting it to the vertices of the triangle (see Figure 11, left). The vertex set $V_{\Gamma}$ is in one-to-one correspondence with $V_{\mathrm{T}} \cup T_{\mathrm{T}}$, and the edge set $E_{\Gamma}$ is in one-to-one correspondence with the set of angles $A_{\mathrm{\top}}$. 

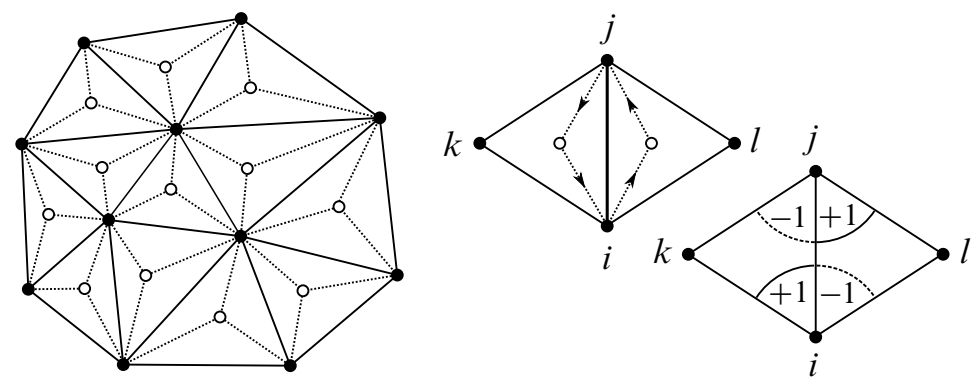

Figure 11: On the left, we have the triangulation $\mathrm{T}$ (solid) and the corresponding graph $\Gamma$ (dotted). On the right, we have the cycle of edges of $\Gamma$ (dashed) that corresponds to an interior edge $i j \in E_{\mathrm{T}}$, and the corresponding tangent vector $v_{i j} \in \mathbb{R}^{A}$ to $C_{\Theta}$.

The tangent space to $C_{\Theta} \subseteq \mathbb{R}^{A}$, which consists of those vectors in $\mathbb{R}^{A}$ that sum to 0 in each triangle and around each vertex, is in one-to-one correspondence with the space of closed edge chains of $\Gamma$ with coefficients in $\mathbb{R}$.

First, assume that $\tilde{\alpha}$ is a critical point of $\left.S_{\mathrm{T}, \lambda}\right|_{C}$. Suppose $i j \in E_{\mathrm{T}}$ is an interior edge and consider the cycle of edges of $\Gamma$ shown on the right in Figure 11. The corresponding tangent vector to $C_{\Theta}$ in $\mathbb{R}^{A}$ is

$$
\begin{gathered}
\frac{\partial}{\partial \alpha_{i l}^{j}}-\frac{\partial}{\partial \alpha_{l j}^{i}}+\frac{\partial}{\partial \alpha_{j k}^{i}}-\frac{\partial}{\partial \alpha_{k i}^{j}} \\
\left(\frac{\partial}{\partial \alpha_{i l}^{j}}-\frac{\partial}{\partial \alpha_{l j}^{i}}+\frac{\partial}{\partial \alpha_{j k}^{i}}-\frac{\partial}{\partial \alpha_{k i}^{j}}\right) S_{\mathrm{T}, \lambda}(\widetilde{\alpha})=-2 \log \left(\frac{\sin \left(\widetilde{\alpha}_{i l}^{j}\right) \sin \left(\widetilde{\alpha}_{j k}^{i}\right)}{\sin \left(\widetilde{\alpha}_{l j}^{i}\right) \sin \left(\widetilde{\alpha}_{k i}^{j}\right)}\right) \\
+\lambda_{i l}-\lambda_{l j}+\lambda_{j k}-\lambda_{k i} .
\end{gathered}
$$

Provided that $\tilde{\alpha}$ is in fact the system of angles of a discrete metric $\tilde{\ell}=e^{\tilde{\lambda} / 2}$, this implies that $\tilde{\ell}$ and $\ell$ are discretely conformally equivalent. It remains to show that $\tilde{\alpha}$ is indeed the system of angles of a discrete metric. Construct such a metric as follows: pick one edge $i j \in E_{\mathrm{T}}$ and choose an arbitrary value for $\tilde{\lambda}_{i j}$. To define $\tilde{\lambda}_{l k}$ for any other edge $l k \in E_{\mathrm{T}}$, connect it to $i j$ by an edge-connected sequence of triangles as shown in Figure 12, let $\gamma$ be the chain of edges of the graph $\Gamma$ as indicated in the figure. Let $w_{\gamma}$ be the corresponding vector in $\mathbb{R}^{A}$ and define

$$
\tilde{\lambda}_{k l}=\tilde{\lambda}_{i j}+d S_{\mathrm{T}, \lambda} \mid \tilde{\alpha}\left(w_{\gamma}\right)+\lambda_{k l}-\lambda_{i j} .
$$

The value of $\tilde{\lambda}_{k l}$ obtained in this way is independent of the choice of triangle chain: another triangle chain connecting $i j$ to $k l$ leads to an edge-chain $\gamma^{\prime}$ such that $\gamma^{\prime}-\gamma$ is a closed edge-chain so that $w_{\gamma^{\prime}}-w_{\gamma} \in \mathbb{R}^{A}$ is tangent to $C_{\Theta}$. Further, $\tilde{\ell}=e^{\tilde{\lambda} / 2}$ is 
a discrete metric with angles $\tilde{\alpha}$ : if $i j$ and $k l$ belong to the same triangle (that is, if the triangle chain consists of only one triangle) then this follows from the sine rule. The general case follows by induction over the length of $\gamma$. So $\tilde{\ell}$ is a solution of Problem 3.1.1.

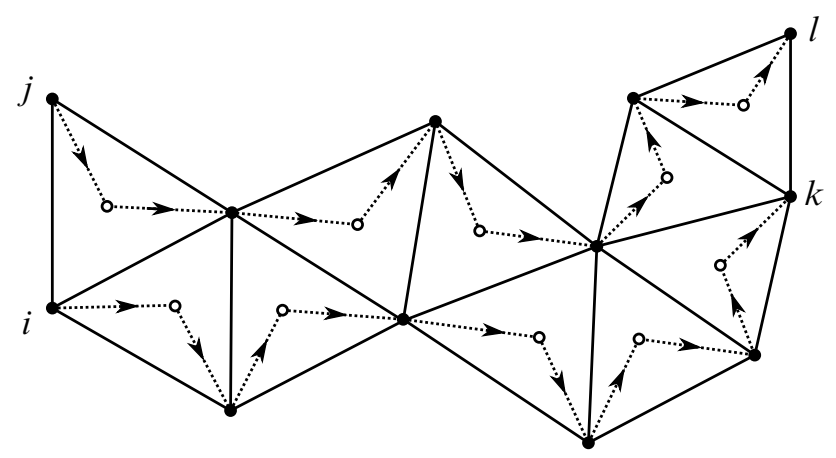

Figure 12: Edge-connected chain of triangles from edge $i j$ to edge $\mathrm{kl}$ of T: the corresponding chain $\gamma$ of $\Gamma$-edges (dotted) consists of the edges of $\Gamma$ opposite the initial edge $i j$, the terminal edge $k l$ and the intermediate connecting edges of the triangle chain.

The converse implication (solution of Problem 3.1.1 implies critical point) follows from the fact that the cycle space of $\Gamma$ is spanned by the cycles corresponding to interior edges of $T$ as shown in Figure 11(right) together with the cycles in $\Gamma$ corresponding to edge-connected triangle sequences as shown in Figure 12 but closed.

Solutions to the more general Problem 3.1.3 (with $\left.u\right|_{V_{0}}=0$ ) are also in one-to-one correspondence with critical points of $S_{\mathrm{T}, \lambda}$. The only difference is that the angle sums are not constrained for vertices in $V_{0}$ :

Proposition 4.3.4 (Second variational principle II) Let $\mathcal{C}$ be a discrete conformal class on $T$ with representative $\ell=e^{\lambda / 2} \in \mathbb{R}^{E}$, let $V=V_{0} \dot{\cup} V_{1}$ be a partition of $V$, let $\Theta \in \mathbb{R}^{V_{1}}$, and define $C_{\Theta}^{\prime} \subseteq \mathbb{R}^{A}$ by

$$
\begin{array}{r}
C_{\Theta}^{\prime}=\left\{\alpha \in \mathbb{R}^{A} \mid \alpha>0, \text { for all } i j k \in T: \alpha_{j k}^{i}+\alpha_{k i}^{j}+\alpha_{i j}^{k}=\pi,\right. \\
\left.\quad \text { for all } i \in V_{1}: \sum_{j k: i j k \in T} \alpha_{j k}^{i}=\Theta_{i}\right\}
\end{array}
$$

Then $\tilde{\alpha} \in C_{\Theta}^{\prime}$ is the angle function of a solution of Problem 3.1.3 with fixed $\left.u\right|_{V_{0}}=0$ if and only if $S_{\mathrm{T}, \lambda}(\widetilde{\alpha})$ is the maximum of the restriction $\left.S_{\mathrm{T}, \lambda}\right|_{C_{\Theta}^{\prime}}$. 
We omit the proof because no essential new ideas are necessary beyond those used in the proof of Proposition 4.3.3.

Remark 4.3.5 It also makes sense to consider critical points of $S_{\mathrm{T}, \lambda}$ under variations of the type shown in Figure 11 alone, disallowing variations corresponding to homologically nontrivial cycles in $\Gamma$. These correspond to discretely conformally equivalent similarity structures, that is, "metrics" which may have global scaling holonomy.

\section{The other side of the theory: Interpretation in terms of hyperbolic geometry}

\subsection{Hyperbolic structure on a euclidean triangulation}

This section deals with the inverse of a construction of Penner [32;11], which equips a hyperbolic manifold with cusps with a piecewise euclidean metric. Here, we construct a natural hyperbolic metric with cusps on any euclidean triangulation.

Consider a euclidean triangle with its circumcircle. If we interpret the interior of the circumcircle as a hyperbolic plane in the Klein model, then the euclidean triangle becomes an ideal hyperbolic triangle, that is, a hyperbolic triangle with vertices at infinity. This construction equips any euclidean triangle (minus its vertices) with a hyperbolic metric. If it is performed on all triangles of a euclidean triangulation $(\mathrm{T}, \ell$ ), then the hyperbolic metrics induced on the individual triangles fit together so $\mathrm{T} \backslash V$ is equipped with a hyperbolic metric with cusps at the vertices. Thus, $\mathrm{T}$ becomes an ideal triangulation of a hyperbolic surface with cusps.

Remark 5.1.1 We will see in Section 5.3 that $\lambda_{i j}$ and $\log \operatorname{lcr}_{i j}$ are Penner coordinates and shear coordinates for this hyperbolic surfaces. It follows that the above construction yields the same surface as a construction described (in terms of length-cross-ratios) by Rivin [36, Section 7].

Theorem 5.1.2 Two euclidean triangulations $(\mathrm{T}, \ell)$ and $(\mathrm{T}, \tilde{\ell})$ with the same combinatorics are discretely conformally equivalent if and only if the hyperbolic metrics with cusps induced by the circumcircles are isometric. Discrete conformal maps are isometries with respect to the induced hyperbolic metrics.

Proof This follows immediately from Theorem 2.6.2 (Section 2.6), because the projective circumcircle-preserving maps between triangles are precisely the hyperbolic isometries. 
Remark 5.1.3 Each discrete conformal structure on $T$ corresponds therefore to a point in the classical Teichmüller space $\mathcal{T}_{g, n}$ of a punctured surface. This explains the dimensional agreement observed in Remark 2.1.2.

Theorem 5.1.2 also suggests a way to extend the concepts of discrete conformal equivalence and discrete conformal maps to triangulations which are not combinatorially equivalent:

Definition 5.1.4 Two euclidean triangulations $(T, \ell)$ and $(\tilde{T}, \tilde{\ell})$, which need not be combinatorially equivalent, are discretely conformally equivalent if they are isometric with respect to the induced hyperbolic metrics with cusps. The corresponding isometries are called discrete conformal maps.

\subsection{Decorated ideal triangles and tetrahedra}

In this section we review some basic facts about ideal triangles and tetrahedra that will be needed in subsequent sections.

All ideal hyperbolic triangles are congruent with respect to the group of hyperbolic isometries. A decorated ideal triangle is an ideal hyperbolic triangle together with a choice of horocycles, one centered at each vertex (see Figure 13).
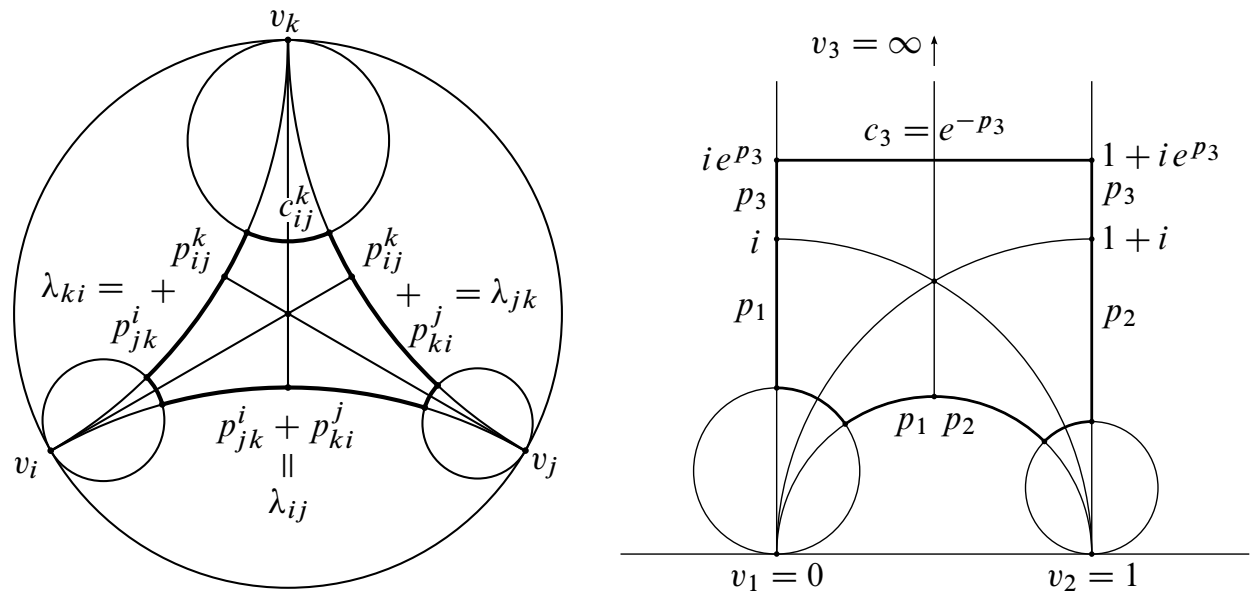

Figure 13: Decorated ideal triangle in the Poincare disk model (left) and in the half-plane model (right)

We denote by $\lambda_{i j}$ the signed distance between the horocycles at vertices $i$ and $j$ as measured along the edge $i j$ and taken negatively if the horocycles intersect. Any triple of real numbers $\left(\lambda_{i j}, \lambda_{j k}, \lambda_{k i}\right) \in \mathbb{R}^{3}$ corresponds to one and only one choice of 
horocycles. Figure 13 shows also the lines of symmetry of the ideal triangle. (They are its heights as well.) We denote the signed distances from their base points to the horocycles by $p_{i j}^{k}$ as shown. Clearly,

$$
\lambda_{i j}=p_{j k}^{i}+p_{k i}^{j}, \quad \lambda_{j k}=p_{k i}^{j}+p_{i j}^{k}, \quad \lambda_{k i}=p_{i j}^{k}+p_{j k}^{i},
$$

so

$$
\begin{aligned}
& p_{i j}^{k}=\frac{1}{2}\left(-\lambda_{i j}+\lambda_{j k}+\lambda_{k i}\right), \\
& p_{j k}^{i}=\frac{1}{2}\left(\lambda_{i j}-\lambda_{j k}+\lambda_{k i}\right), \\
& p_{k i}^{j}=\frac{1}{2}\left(\lambda_{i j}+\lambda_{j k}-\lambda_{k i}\right) .
\end{aligned}
$$

Lemma 5.2.1 (Penner [32]) The length $c_{i j}^{k}$ of the arc of the horocycle centered at $v_{k}$ that is contained in an ideal triangle $v_{i} v_{j} v_{k}$ as shown in Figure 13 (left) is

$$
c_{i j}^{k}=e^{-p_{i j}^{k}}=e^{\left(\lambda_{i j}-\lambda_{j k}-\lambda_{k i}\right) / 2} .
$$

Proof See Figure 13 (right), which shows the ideal triangle in the half-plane model. Recall that in the half-plane model, the hyperbolic plane is represented by $\{z \in \mathbb{C} \mid$ $\operatorname{Im} z>0\}$ with metric $d s=\frac{1}{\operatorname{Im} z}|d z|$.

Remark 5.2.2 Together with Proposition 5.3.2 of the next section, this provides a geometric interpretation for the auxiliary parameters $c_{j k}^{i}$ introduced in Section 2.4.

Not all ideal tetrahedra are isometric. There is a complex 1-parameter family of them, the parameter being the complex cross-ratio of the vertices in the infinite boundary of hyperbolic 3-space. A decorated ideal tetrahedron is an ideal hyperbolic tetrahedron together with a choice of horospheres centered at the vertices. Figure 14 shows a decorated ideal tetrahedron, truncated at its horospheres, in the half-space model. Again, we denote the signed distances between the horospheres by $\lambda_{i j}$.

The intrinsic geometry of a horosphere in hyperbolic space is euclidean. So the intersection of the tetrahedron with the horosphere centered at, say, $v_{l}$ is a euclidean triangle with side lengths $c_{i j}^{l}, c_{j k}^{l}, c_{k i}^{l}$ determined by Lemma 5.2.1. One easily deduces the following lemma.

Lemma 5.2.3 Six real numbers $\lambda_{i j}, \lambda_{j k}, \lambda_{k i}, \lambda_{i l}, \lambda_{j l}, \lambda_{k l}$ are the signed distances between horospheres of a decorated ideal tetrahedron as shown in Figure 14 (which is then unique) if and only if $c_{i j}^{l}, c_{j k}^{l}, c_{k i}^{l}$ determined by Lemma 5.2.1 satisfy the triangle inequalities. 


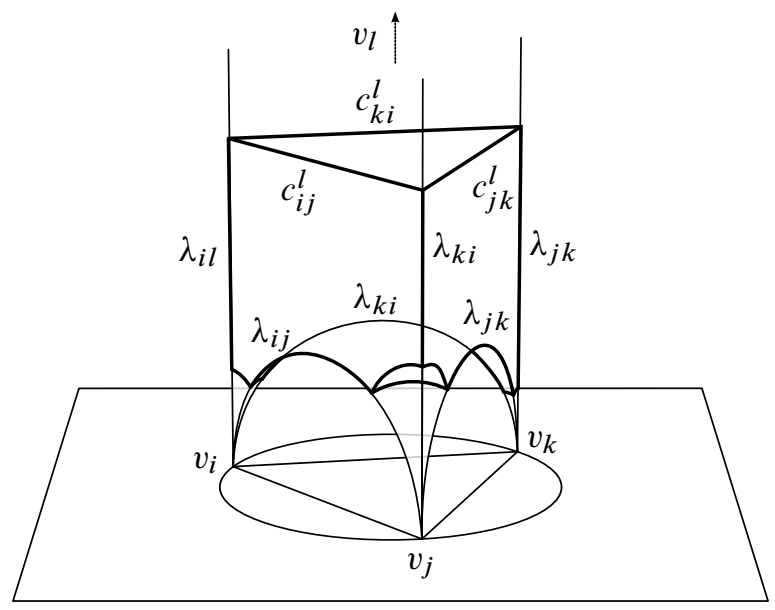

Figure 14: Decorated ideal tetrahedron in the half-space model

So the six parameters $\lambda$ determine the congruence class of the ideal tetrahedron ( 2 real parameters) and the choice of horospheres ( 4 parameters).

Note that the angles of the euclidean triangles in which the tetrahedron intersects the four horospheres are the dihedral angles of the tetrahedron. This implies that the dihedral angles sum to $\pi$ at each vertex, and further, that the dihedral angles at opposite edges are equal. The space of ideal tetrahedra is therefore parametrized by three dihedral angles $\alpha_{i j}=\alpha_{k l}, \alpha_{j k}=\alpha_{i l}, \alpha_{k i}=\alpha_{j l}$ satisfying $\alpha_{i l}+\alpha_{j l}+\alpha_{k l}=\pi$.

\subsection{Penner coordinates and shear coordinates}

In Section 5.1, we equipped a euclidean triangulation $(T, \ell)$ with a hyperbolic cusp metric that turns it into an ideal hyperbolic triangulation. In this section, we will identify the logarithmic edge lengths $\lambda$ (see Equation (2-2)) with the Penner coordinates [32] and the logarithmic length-cross-ratios log lcr (see Equation (2-4)) with the shear coordinates (see Fock [12] and Thurston [43]) for this ideal triangulation. (The handbook [30] is a good reference for the pertinent aspects of Teichmüller theory.)

Warning Our notation differs from Penner's in a potentially confusing way. His "lambda-lengths" are $\sqrt{2} e^{\lambda / 2}=\sqrt{2} \ell$ in our notation. Our $\lambda \mathrm{s}$ are the signed hyperbolic distances between horocycles.

Since the sides of an ideal hyperbolic triangle are complete geodesics, there is a oneparameter family of ways to glue two sides together. Penner coordinates and shear 
coordinates can be seen as two ways to describe how ideal triangles are glued together along their edges to form a hyperbolic surface with cusps.

Suppose $T$ is a triangulated surface and $\lambda \in \mathbb{R}^{E}$. For each triangle $i j k \in T$, take the decorated ideal triangle with horocycle distances $\lambda_{i j}, \lambda_{j k}, \lambda_{k i}$, and glue them so that the horocycles fit together (see Figure 15(left)).
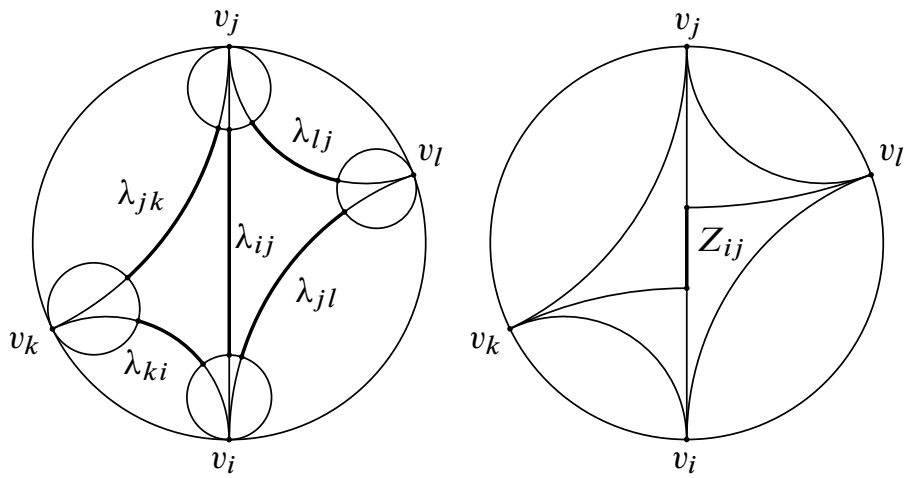

Figure 15: Penner coordinates (left) and shear coordinates (right)

The result is a hyperbolic surface with cusps at the vertices, together with a particular choice of horocycles centered at the cusps. In this way, the Penner coordinates $\lambda$ parametrize the decorated Teichmüller space, that is, the space of hyperbolic cusp metrics on a punctured surface (modulo isotopy) with horocycles centered at the cusps. The shear coordinates represent another way to prescribe how ideal triangles are glued, for which no choice of horospheres is necessary. The shear coordinate $Z$ on an interior edge of an ideal triangulation is the signed distance of the base points of the heights from the opposite vertices (see Figure 15(right)). The following relation between Penner coordinates and shear coordinates is well known.

Lemma 5.3.1 If $\lambda \in \mathbb{R}^{E}$ are the Penner coordinates for an ideal triangulation with a particular choice of horocycles, then the shear coordinates $Z \in \mathbb{R}^{E_{\text {int }}}$ are

$$
Z_{i j}=\frac{1}{2}\left(\lambda_{i l}-\lambda_{l j}+\lambda_{j k}-\lambda_{k i}\right)
$$

where $k$ and $l$ are the vertices opposite edge $i j$ as in Figure 15.

Proof The claim follows from $Z_{i j}=p_{k i}^{j}-p_{i l}^{j}$ and (5-1).

Proposition 5.3.2 Let $(T, \ell)$ be a euclidean triangulation. The shear coordinates $Z \in \mathbb{R}^{E_{\text {int }}}$ for the corresponding ideal triangulation (see Section 5.1) are

$$
Z_{i j}=\log \operatorname{lcr}_{i j}
$$


(see Equation (2-4)). Thus, for a suitable choice of horocycles, the Penner coordinates $\lambda \in \mathbb{R}^{E}$ are given by Equation (2-2).

Proof Consider an interior edge $i j \in E$ between triangles $i j k$ and $j i l$. Without loss of generality, we may assume that the triangles have a common circumcircle. For otherwise we can change $\ell$ discretely conformally so that this holds, and this changes neither $\operatorname{lcr}_{i j}$ nor the hyperbolic cusp metric on $T$. We may further assume that $i j$ is a diameter of the common circumcircle. For otherwise we may apply a projective transformation that maps the circle onto itself so that this holds. This is an isometry with respect to the hyperbolic metric of the Klein model, and it is a discrete conformal map of the quadrilateral formed by the two triangles. We arrive at the situation shown in Figure 16 in the Klein model.

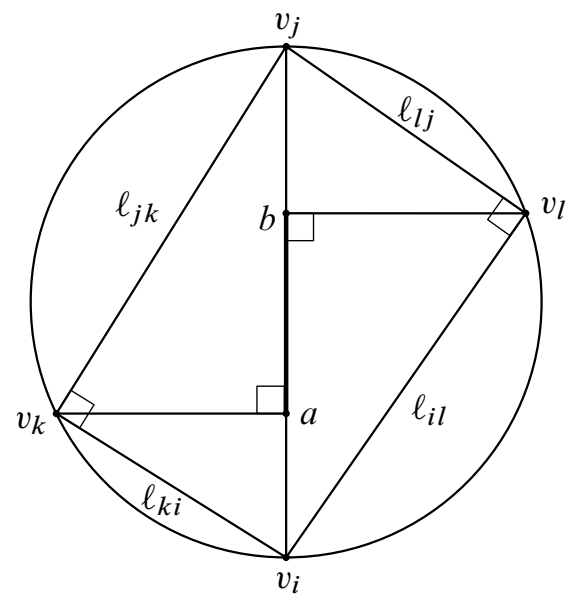

Figure 16: Shear and length-cross-ratio (see proof of Proposition 5.3.2)

The hyperbolic heights are also the euclidean heights, and in the hyperbolic metric of the Klein model, the distance between their base points $a$ and $b$ is

$$
Z_{i j}=\frac{1}{2} \log \frac{\left|a v_{j}\right|\left|b v_{i}\right|}{\left|a v_{i}\right|\left|b v_{j}\right|}
$$

where $|x y|$ denotes the euclidean distance between $x$ and $y$. Since by elementary euclidean geometry

$$
\frac{\left|a v_{j}\right|}{\left|a v_{i}\right|}=\frac{\ell_{j k}^{2}}{\ell_{k i}{ }^{2}} \text { and } \quad \frac{\left|b v_{i}\right|}{\left|b v_{j}\right|}=\frac{\ell_{l j}^{2}}{\ell_{i l^{2}}{ }^{2}},
$$

this implies Equation (5-2). Now Lemma 5.3.1 implies the statement about Penner coordinates. 


\subsection{Ideal hyperbolic polyhedra with prescribed intrinsic metric}

The discrete conformal mapping problems described in Section 3 are equivalent to problems involving the polyhedral realization of surfaces with hyperbolic cusp metrics, like the following.

Problem 5.4.1 Given an ideal triangulation $T$ of a punctured sphere equipped with a hyperbolic metric with cusps, find an isometric embedding of $T$ as ideal hyperbolic polyhedron in $H^{3}$. The polyhedron is not required to be convex, but it is required that the edges of the polyhedron are edges of $\mathrm{T}$.

Theorem 5.4.2 For any vertex $l$ of $\mathrm{T}$, Problem 5.4.1 has at most one solution that is star-shaped with respect to $l$.

Problem 5.4.1 is equivalent to (a special case of) Problem 3.1.3, so Theorem 5.4.2 follows from Theorem 3.1.4. Indeed, to solve Problem 5.4.1 one may proceed as follows. Let $\lambda \in \mathbb{R}^{E}$ be the Penner coordinates for the ideal triangulation $\mathrm{T}$, and let $\ell=e^{\lambda / 2}$. Choose a vertex $l$ of $\mathrm{T}$ and let the triangulation $\mathrm{T}^{\prime}$ be $\mathrm{T}$ minus the open star of $l$. Solve Problem 3.1.3 for $\mathrm{T}^{\prime}$, prescribing $\Theta_{i}=2 \pi$ if $i$ is an interior vertex and $u_{i}=-\lambda_{i l}$ if $i$ is a boundary vertex. Suppose a solution $u \in \mathbb{R}^{V^{\prime}}$ exists. This leads to a flat triangulation $\left(T^{\prime}, \tilde{\ell}\right)$. Suppose further that $\left(T^{\prime}, \tilde{\ell}\right)$ does not overlap with itself when developed in the plane. For each triangle $i j k$ of $\mathrm{T}^{\prime}$, construct the decorated ideal tetrahedron (see Section 5.2) with horosphere-distances $\lambda_{i j}, \lambda_{j k}, \lambda_{k i}$, and $-u_{i},-u_{j},-u_{k}$ as shown in Figure 17.

They exist by Lemma 5.2.3, because by Lemma 5.2.1, the intersection of the ideal tetrahedron with the horosphere centered at the vertex opposite $i j k$ is the euclidean triangle with side lengths $\tilde{\ell}_{i j}, \tilde{\ell}_{j k}, \tilde{\ell}_{k i}$ as shown in the figure. Hence, all these ideal tetrahedra fit together to form a solution of Problem 5.4.1 that is star-shaped with respect to $l$.

Conversely, any solution of Problem 5.4.1 that is star-shaped with respect to $l$ yields a solution without self-overlap of the corresponding instance of Problem 3.1.3.

Remark 5.4.3 Note the similarity with the procedure for mapping to a sphere described in Section 3.2.

Numerous variations of Problem 5.4.1 can be treated in similar fashion. We mention only the following.

Problem 5.4.4 Given an ideal triangulation $T$ of a punctured torus equipped with a hyperbolic metric with cusps, find an isometric embedding of the universal cover 


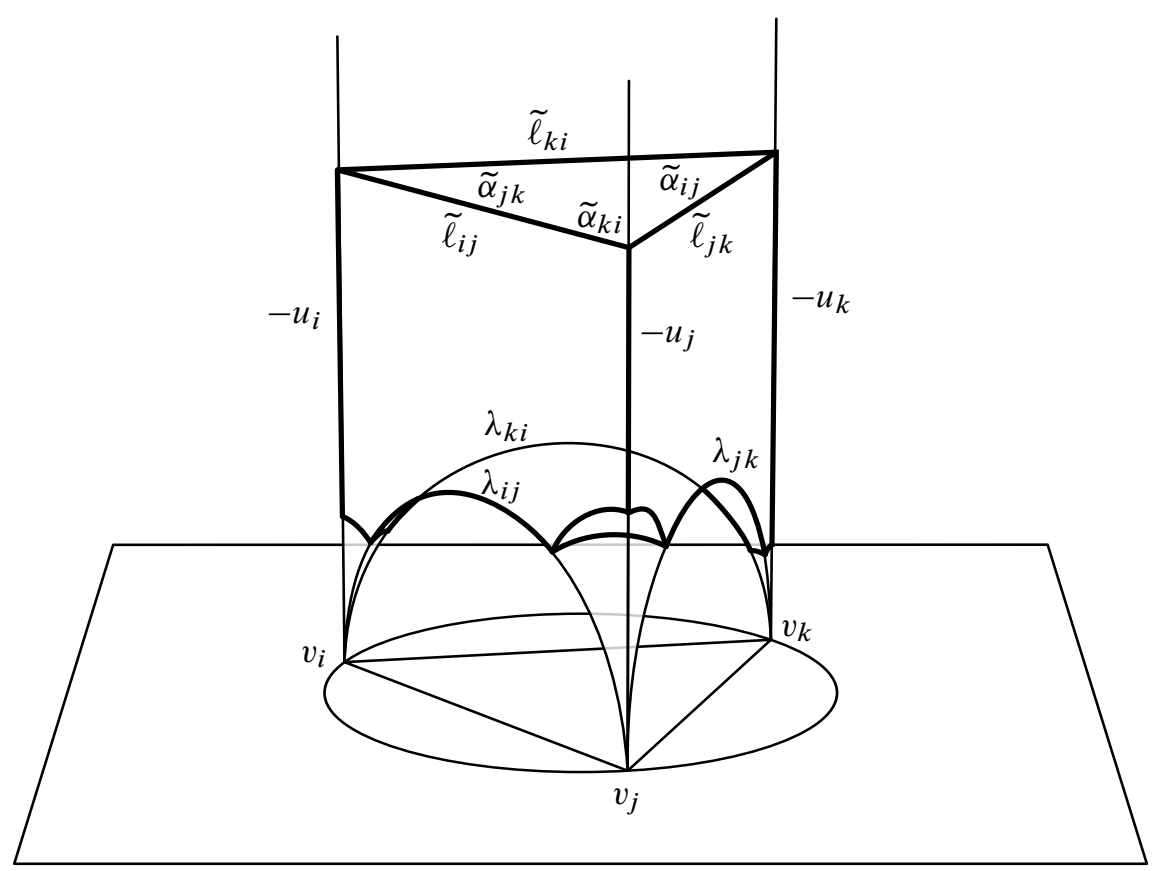

Figure 17: Discretely conformally flattening a euclidean triangulation is equivalent to constructing an ideal polyhedron with prescribed hyperbolic cusp metric.

of $T$ as an ideal polyhedron in $H^{3}$ that is symmetric with respect to an action of the fundamental group of $\mathrm{T}$ by parabolic isometries. The polyhedron is not required to be convex, but it is required that the polyhedron is star-shaped with respect to the ideal fixed point of the parabolic isometries and that the edges of the polyhedron are edges of $T$.

Theorem 5.4.5 If Problem 5.4.4 has a solution, it is unique.

\subsection{The variational principles and hyperbolic volume}

The connection with hyperbolic polyhedra elucidates the nature and origin of the variational principles for discrete conformal maps (Propositions 4.1.3, 4.3.3 and 4.3.4). In this section, we will indicate how to derive these variational principles from Milnor's equation for the volume of an ideal tetrahedron and Schläfli's formula.

Milnor $[27 ; 28]$ showed that the volume of an ideal tetrahedron with dihedral angles $\alpha, \beta, \gamma$ is $V(\alpha, \beta, \gamma)$ as defined by Equation (4-11). Schläfli's differential volume 
formula (more precisely, Milnor's generalization which allows for ideal vertices [29]) says that its derivative is

$$
d V=-\frac{1}{2} \sum \lambda_{i j} d \alpha_{i j}
$$

where the sum is taken over the six edges $i j, \lambda_{i j}$ is the signed distance between horospheres centered at the vertices $i$ and $j$, and $\alpha_{i j}$ is the interior dihedral angle. (The choice of horospheres does not matter because the dihedral angle sum at a vertex is constant; see Section 5.2.)

Using the correspondence between ideal tetrahedra and euclidean triangles, the volume function $V$ can be reinterpreted as a function of the angles of a euclidean triangle, whose derivatives $((\partial / \partial \alpha)-(\partial / \partial \beta)) V$, etc, are logarithmic ratios of the sides. This is the essential property of $V$ used in the second variational principle (Propositions 4.3.3 and 4.3.4).

Now define

$$
\widehat{V}\left(\lambda_{12}, \lambda_{23}, \lambda_{31}, \lambda_{14}, \lambda_{24}, \lambda_{34}\right)=\frac{1}{2} \sum_{i j} \alpha_{i j} \lambda_{i j}+V\left(\alpha_{14}, \alpha_{24}, \alpha_{34}\right),
$$

where the dihedral angles $\alpha_{12}=\alpha_{34}, \alpha_{23}=\alpha_{14}, \alpha_{31}=\alpha_{24}$ of the decorated tetrahedron are considered as functions of the $\lambda_{i j}$. (They are the angles in a euclidean triangle with side lengths $e^{\left(\lambda_{12}-\lambda_{14}-\lambda_{24}\right) / 2}, e^{\left(\lambda_{23}-\lambda_{24}-\lambda_{34}\right) / 2}, e^{\left(\lambda_{31}-\lambda_{34}-\lambda_{14}\right) / 2}$; see Section 5.2.) Then, by Equation (5-3),

$$
d \widehat{V}=\frac{1}{2} \sum \alpha_{i j} d \lambda_{i j}
$$

This implies Proposition 4.1.2 on the partial derivatives of $E_{\mathrm{T}, \Theta, \lambda}$, and therefore Proposition 4.1.3 (the first variational principle), because using (5-4) (and (2-3)) we can rewrite (4-1) as

(5-6) $E_{\mathrm{T}, \Theta, \lambda}(u)=\sum_{i j k \in T} 2 \widehat{V}\left(\lambda_{i j}, \lambda_{j k}, \lambda_{k i},-u_{i},-u_{j},-u_{k}\right)-\sum_{i j \in E} \Phi_{i j} \lambda_{i j}+\sum_{i \in V} \Theta_{i} u_{i}$,

where

$$
\Phi_{i j}= \begin{cases}\pi & \text { if } i j \text { is an interior edge, } \\ \pi / 2 & \text { if } i j \text { is a boundary edge. }\end{cases}
$$

(See also Figure 17.) 


\section{The discrete conformal equivalence of hyperbolic triangu- lations}

\subsection{Definition and variational principle}

In Section 5.5 we derived the first variational principle for discrete conformal maps from Milnor's equation for the volume of an ideal tetrahedron and Schläfli's formula. A straightforward modification of this derivation leads to a companion theory of discrete conformality for hyperbolic triangulations. This makes it possible, for example, to construct discretely conformal uniformizations of higher-genus surfaces as shown in Figure 18.
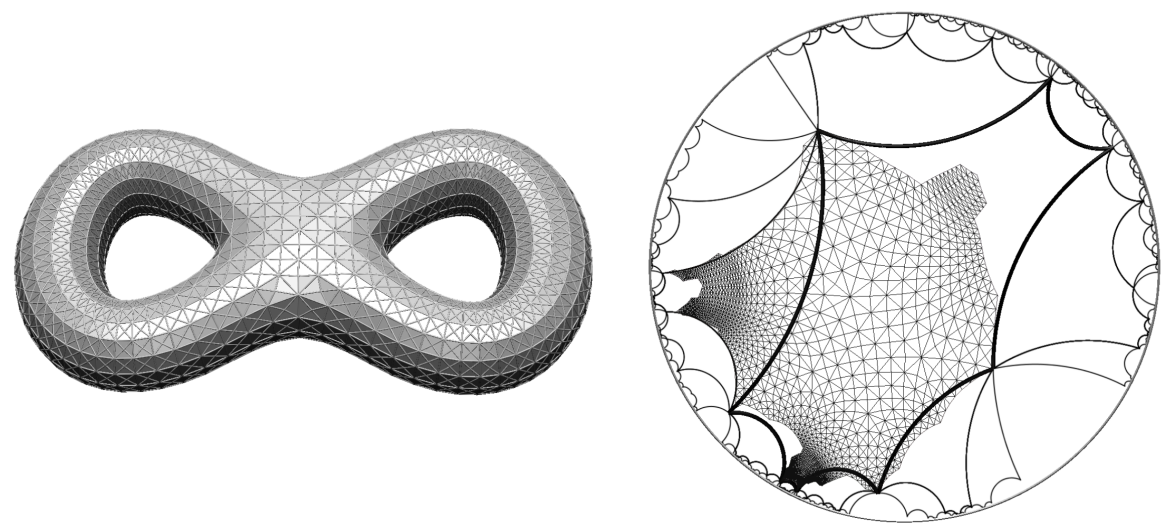

Figure 18: Discretely conformal uniformization of a genus-two surface

We will present the basic theory in this section, and show how to derive it by hyperbolic volume considerations in the next.

Suppose $T$ is a surface triangulation and $\ell \in \mathbb{R}_{>0}^{E}$ is a discrete metric, that is, a realvalued function on the set of edges that satisfies all triangle inequalities. Then there is not only a euclidean triangulation $(T, \ell)$ with these edge lengths. One can equally construct hyperbolic triangles $i j k$ with hyperbolic side lengths $\ell_{i j}, \ell_{j k}, \ell_{k i}$ and glue them together. The result is a hyperbolic triangulation which we denote by $(\mathrm{T}, \ell)_{h}$.

Definition 6.1.1 Two combinatorially equivalent hyperbolic triangulations, $(T, \ell)_{h}$ and $(\mathrm{T}, \tilde{\ell})_{h}$, are discretely conformally equivalent if the discrete metrics $\ell$ and $\tilde{\ell}$ are related by

$$
\sinh \frac{\tilde{\ell}_{i j}}{2}=e^{\left(u_{i}+u_{j}\right) / 2} \sinh \frac{\ell_{i j}}{2}
$$

for some function $u: V \rightarrow \mathbb{R}$. 
Thus, in the hyperbolic version of the theory, (2-2) is replaced by

$$
\lambda=2 \log \sinh \frac{\ell}{2},
$$

so that in terms of $\lambda$ and $\tilde{\lambda}$, the relation (6-1) is again equivalent to (2-3). The role of $E_{\mathrm{T}, \Theta, \lambda}(u)$ is played by the function

$$
E_{\mathrm{T}, \Theta, \lambda}^{h}(u)=\sum_{i j k \in T} 2 \hat{V}_{h}\left(\lambda_{i j}, \lambda_{j k}, \lambda_{k i},-u_{i},-u_{j},-u_{k},\right)+\sum_{i \in V} \Theta_{i} u_{i},
$$

where

$$
\begin{aligned}
& 2 \hat{V}_{h}\left(\lambda_{12}, \lambda_{23}, \lambda_{31}, \lambda_{1}, \lambda_{2}, \lambda_{3}\right) \\
& =\alpha_{1} \lambda_{1}+\alpha_{2} \lambda_{2}+\alpha_{3} \lambda_{3}+\alpha_{12} \lambda_{12}+\alpha_{23} \lambda_{23}+\alpha_{31} \lambda_{31} \\
& +\pi\left(\alpha_{1}\right)+\pi\left(\alpha_{2}\right)+\pi\left(\alpha_{3}\right)+
\end{aligned}
$$

and $\alpha_{1}, \alpha_{2}, \alpha_{3}$ are the angles in a hyperbolic triangle with side lengths

$$
\begin{gathered}
\tilde{\ell}_{23}=2 \operatorname{arsinh}\left(e^{\left(\lambda_{23}-\lambda_{2}-\lambda_{3}\right) / 2}\right), \\
\tilde{\ell}_{31}=2 \operatorname{arsinh}\left(e^{\left(\lambda_{31}-\lambda_{3}-\lambda_{1}\right) / 2}\right), \\
\tilde{\ell}_{12}=2 \operatorname{arsinh}\left(e^{\left(\lambda_{12}-\lambda_{1}-\lambda_{2}\right) / 2}\right), \\
\alpha_{23}=\frac{1}{2}\left(\pi+\alpha_{1}-\alpha_{2}-\alpha_{3}\right), \\
\alpha_{31}=\frac{1}{2}\left(\pi-\alpha_{1}+\alpha_{2}-\alpha_{3}\right), \\
\alpha_{12}=\frac{1}{2}\left(\pi-\alpha_{1}-\alpha_{2}+\alpha_{3}\right) .
\end{gathered}
$$

Thus, $\widehat{V}_{h}$ is defined only on the domain where $\tilde{\ell}_{12}, \tilde{\ell}_{23}, \tilde{\ell}_{31}$ satisfy the triangle inequalities. However, exactly as in the case of $E_{\mathrm{T}, \Theta, \lambda}(u)$, we can extend the domain of definition of $E_{\mathrm{T}, \Theta, \lambda}^{h}(u)$ to the whole of $\mathbb{R}^{V}$ :

Proposition 6.1.2 Extend the domain of definition of $E_{\mathrm{T}, \Theta, \lambda}^{h}(u)$ to $\mathbb{R}^{V}$ by declaring the angles in "broken" triangles to be $0,0, \pi$, respectively. The resulting function is continuously differentiable on $\mathbb{R}^{V}$.

Proof See Section 6.2.

Remark 6.1.3 To compute the angles $\alpha, \beta, \gamma$ in a hyperbolic triangle with side lengths $a, b, c$, one can use, for example, the hyperbolic cosine rule or the hyperbolic 
half-angle formula

$$
\tan \left(\frac{\alpha}{2}\right)=\sqrt{\frac{\sinh ((a-b+c) / 2) \sinh ((a+b-c) / 2)}{\sinh ((-a+b+c) / 2) \sinh ((a+b+c) / 2)}} .
$$

Proposition 6.1.4 Let $\ell \in \mathbb{R}^{E}$, let $\lambda$ be defined by (6-2), and suppose $u \in \mathbb{R}^{V}$ is in the domain where $\tilde{\ell}$ defined by (6-1) satisfies all triangle inequalities. Then the partial derivative of $E_{\mathrm{T}, \Theta, \lambda}^{h}$ with respect to $u_{i}$ is

$$
\frac{\partial}{\partial u_{i}} E_{\mathrm{T}, \Theta, \lambda}^{h}=\Theta_{i}-\sum_{j k: i j k \in T} \tilde{\alpha}_{j k}^{i},
$$

where $\tilde{\alpha}$ are the angles in the hyperbolic triangulation $(\mathrm{T}, \tilde{\ell})_{h}$, and the sum is taken over all angles around vertex $i$.

Proof See Section 6.2.

Proposition 6.1.5 The function $E_{\mathrm{T}, \Theta, \lambda}^{h}(u)$ is convex on $\mathbb{R}^{V}$ and locally strictly convex in the domain where $\tilde{\ell}$ defined by (6-1) satisfies all triangle inequalities.

Proof See Section 6.2.

Consider the discrete conformal mapping problems for hyperbolic triangulations that are analogous to those for euclidean triangulations described in Section 3. Propositions 6.1.4 and 6.1.5 imply the following hyperbolic version of Theorem 3.1.4.

Theorem 6.1.6 If the discrete mapping problems for hyperbolic triangulations have a solution, it is unique and can be found by minimizing $E_{\mathrm{T}, \Theta, \lambda}^{h}(u)$.

The following relatively simple explicit formula for the second derivative facilitates the numerical minimization of $E_{\mathrm{T}, \Theta, \lambda}^{h}$.

Proposition 6.1.7 The second derivative of $E_{\mathrm{T}, \Theta, \lambda}^{h}$ at $u$ is

$$
\begin{aligned}
\sum_{i, j \in V} \frac{\partial^{2} E_{\mathrm{T}, \Theta, \lambda}^{h}}{\partial u_{i} \partial u_{j}} d u_{i} d u_{j} \\
\quad=\frac{1}{2} \sum_{i j \in E} w_{i j}(u)\left(\left(d u_{i}-d u_{j}\right)^{2}+\tanh ^{2}\left(\frac{\tilde{\ell}_{i j}}{2}\right)\left(d u_{i}+d u_{j}\right)^{2}\right),
\end{aligned}
$$

with $\tilde{\ell}$ defined by (6-1) and

$$
w_{i j}(u)=\frac{1}{2}\left(\cot \left(\frac{1}{2}\left(\pi-\widetilde{\alpha}_{j k}^{i}-\widetilde{\alpha}_{k i}^{j}+\widetilde{\alpha}_{i j}^{k}\right)\right)+\cot \left(\frac{1}{2}\left(\pi-\widetilde{\alpha}_{i l}^{j}-\widetilde{\alpha}_{l j}^{i}+\widetilde{\alpha}_{j i}^{l}\right)\right)\right)
$$


for interior edges $i j$ with opposite vertices $k$ and $l$ if $\tilde{\ell}$ satisfies the triangle inequalities for $i j k$ and $j i l$ (so that the corresponding angles $\tilde{\alpha}$ are positive and smaller than $\pi$ ). If $i j$ is a boundary edge, there is only one cotangent term. For "broken" triangles, replace the three corresponding cotangent terms with 0 .

We omit the proof, which consists of a lengthy but elementary calculation.

Remark 6.1.8 When are a euclidean and a hyperbolic triangulation discretely conformally equivalent? We propose the following definition: a euclidean triangulation $(\mathrm{T}, \ell)$ and a hyperbolic triangulation $(\mathrm{T}, \tilde{\ell})_{h}$ are discretely conformally equivalent if $\ell$ and $\tilde{\ell}$ are related by

$$
\sinh \frac{\tilde{\ell}_{i j}}{2}=e^{\left(u_{i}+u_{j}\right) / 2} \ell_{i j}
$$

for some function $u \in \mathbb{R}^{V}$.

This is based on the following interpretation of (6-2) and (2-3). Consider the hyperboloid model of the hyperbolic plane, $H^{2}=\left\{x \in \mathbb{R}^{2,1} \mid\langle x, x\rangle=-1, x_{3}>0\right\}$, where $\langle\cdot, \cdot\rangle$ denotes the indefinite scalar product $\langle x, y\rangle=x_{1} y_{1}+x_{2} y_{2}-x_{3} y_{3}$, and the hyperbolic distance $d_{h}(x, y)$ between two points $x, y \in H^{2}$ satisfies

$$
\cosh d_{h}(x, y)=-\langle x, y\rangle .
$$

This implies

$$
\|x-y\|_{h}=2 \sinh \left(\frac{1}{2} d_{h}(x, y)\right),
$$

where we have $\|v\|_{h}=\sqrt{\langle v, v\rangle}$. To every hyperbolic triangle in $H^{2}$ with sides of length $\ell_{12}, \ell_{23}, \ell_{31}$, there corresponds a secant triangle in $\mathbb{R}^{2,1}$ whose sides are the straight line segments in $\mathbb{R}^{2,1}$ connecting the vertices. Their lengths, as measured by $\|\cdot\|_{h}$, are therefore $2 \sinh \left(\ell_{i j} / 2\right)$.

Note that the following statements are equivalent.

(i) The restriction of the indefinite scalar product $\langle\cdot, \cdot\rangle$ of $\mathbb{R}^{2,1}$ to the affine plane of the secant triangle is positive definite and therefore induces a euclidean metric on that plane.

(ii) The side lengths of the secant triangle satisfy the triangle inequalities.

(iii) The circumcircle of the hyperbolic triangle is a proper circle. (The circumcircle of a hyperbolic triangle is either a proper circle or a horocycle or a curve of constant distance from a geodesic.) 
(The analogous statement for the secant triangles of decorated ideal hyperbolic triangles is Penner's Lemma 2.2 [32].)

Now let $(\mathrm{T}, \ell)_{h}$ and $(\mathrm{T}, \tilde{\ell})_{h}$ be two hyperbolic triangulations. The edge lengths of the secant triangles of $(\mathrm{T}, \ell)_{h}$ are $2 e^{\lambda / 2}$ with $\lambda$ defined by (6-2), and similarly for $(\mathrm{T}, \tilde{\ell})_{h}$. We have that $(\mathrm{T}, \ell)_{h}$ and $(\mathrm{T}, \tilde{\ell})_{h}$ are discretely conformally equivalent if and only if $\ell^{\prime}=e^{\lambda / 2}$ and $\tilde{\ell}^{\prime}=e^{\tilde{\lambda} / 2}$ are related by (2-1), that is, related like discrete metrics of discretely conformally equivalent euclidean triangulations.

\subsection{Derivation by volume considerations}

The theory of discrete conformal equivalence for hyperbolic triangulations is based on volume considerations for the type of polyhedron shown in Figure 19.

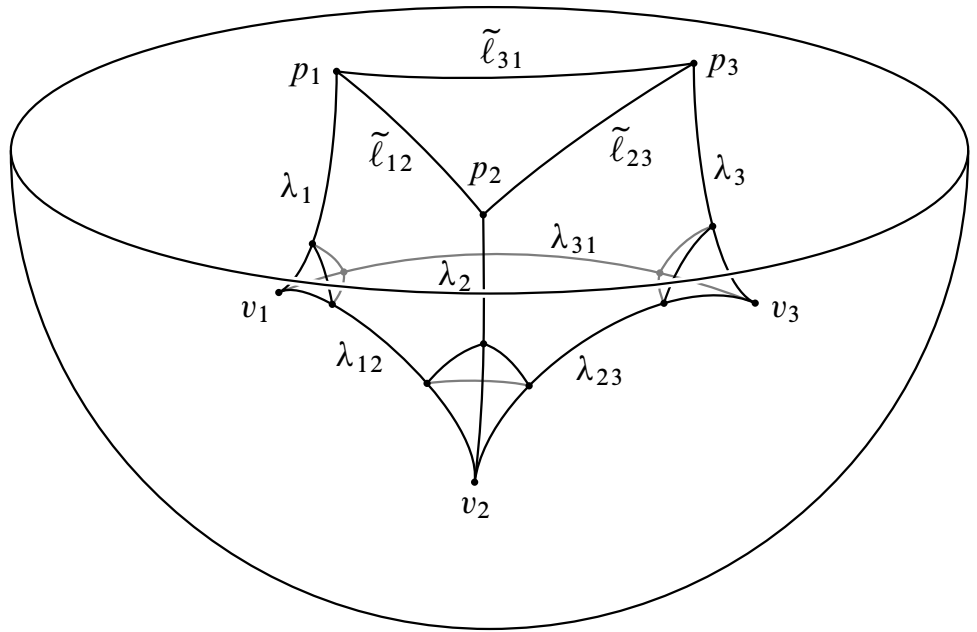

Figure 19: The polyhedral building block (shown in the Poincaré ball model) used to derive the theory of discrete conformal equivalence of hyperbolic triangulations

From the vertices $p_{1}, p_{2}, p_{3}$ of a triangle in hyperbolic 3-space, three rays run orthogonally to the plane of the triangle until they intersect the infinite boundary in the ideal points $v_{1}, v_{2}, v_{3}$. The convex hull of these six points is a prism with three ideal vertices and right dihedral angles at the base triangle $p_{1} p_{2} p_{3}$. Let the dihedral angles at the three rays from $p_{1}, p_{2}, p_{3}$ be $\alpha_{1}, \alpha_{2}, \alpha_{3}$. Since the dihedral angles sum to $\pi$ at the ideal vertices, the dihedral angles $\alpha_{12}, \alpha_{23}, \alpha_{31}$ at edges $v_{1} v_{2}, v_{2} v_{3}$, $v_{3} v_{1}$ satisfy (6-6). Let $\tilde{\ell}_{i j}$ be the lengths of the finite edges, and let $\lambda_{i}$ and $\lambda_{i j}$ be the lengths of the infinite edges truncated at some horospheres centered at the ideal vertices $v_{i}$, as shown in Figure 19. 
Lemma 6.2.1 (Leibon [22]) The (truncated) edge lengths of the prism shown in Figure 19 are related by (6-5).

Proof We consider the case when $\lambda_{1}=\lambda_{2}=\lambda_{3}=0$ (that is, when the truncating horospheres touch the base plane in $\left.p_{1}, p_{2}, p_{3}\right)$, from which the general case follows easily.
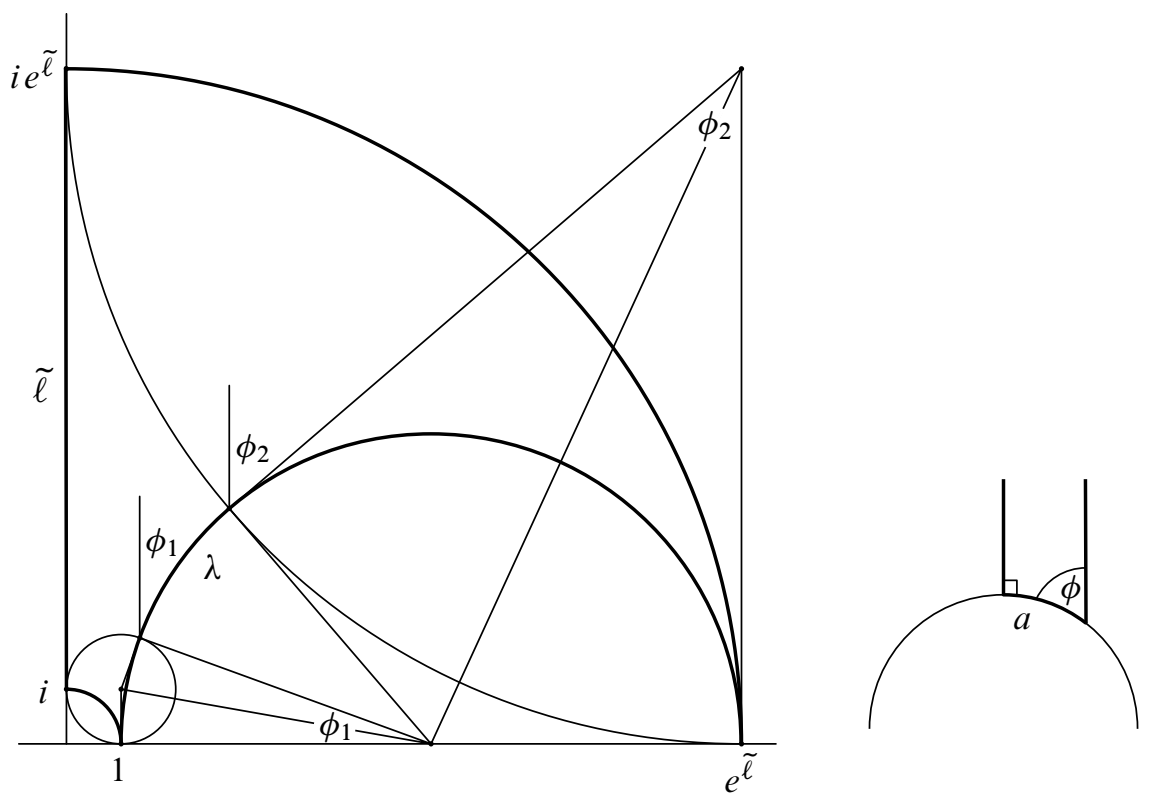

Figure 20: On the left, we have the proof of Lemma 6.2.1. On the right, the angle $\phi$ and the side length $a$ in a right angled hyperbolic triangle with an ideal vertex satisfy the equation $a=\log \cot (\phi / 2)$.

Figure 20(left) shows one of the side quadrilaterals of the prism in the half-plane model. We will show that

$$
\lambda=2 \log \sinh \left(\frac{\tilde{\ell}}{2}\right),
$$

which proves this special case. We have

$$
\phi_{1}=2 \operatorname{arccot}\left(\frac{e^{\tilde{\ell}}-1}{2}\right), \quad \phi_{2}=2 \operatorname{arccot}\left(\frac{2 e^{\tilde{\ell}}}{e^{\tilde{\ell}}-1}\right) .
$$

The equation for the "angle of parallelity" (see Figure 20(right)) implies that

$$
\lambda=\log \cot \left(\phi_{1} / 2\right)-\log \cot \left(\phi_{2} / 2\right),
$$

and hence (6-10) holds. 
The volume of the polyhedron shown in Figure 19 is

$$
\begin{aligned}
V_{h}\left(\alpha_{1}, \alpha_{2}, \alpha_{3}\right)=\frac{1}{2}\left(\pi\left(\alpha_{1}\right)\right. & +\pi\left(\alpha_{2}\right)+\pi\left(\alpha_{3}\right)+\pi\left(\alpha_{12}\right) \\
& \left.+\pi\left(\alpha_{23}\right)+\pi\left(\alpha_{31}\right)+\pi\left(\frac{1}{2}\left(\pi-\alpha_{1}-\alpha_{2}-\alpha_{3}\right)\right)\right) .
\end{aligned}
$$

This was shown by Leibon [22], who also showed that the volume function $V_{h}$ is strictly concave on its domain of definition,

$$
\left\{\left(\alpha_{1}, \alpha_{2}, \alpha_{3}\right) \in \mathbb{R}^{3} \mid \alpha_{1}>0, \alpha_{2}>0, \alpha_{3}>0, \alpha_{1}+\alpha_{2}+\alpha_{3}<\pi\right\} .
$$

By Schläfli's formula,

$$
d V_{h}=-\frac{1}{2}\left(\lambda_{1} d \alpha_{1}+\lambda_{2} d \alpha_{2}+\lambda_{3} d \alpha_{3}+\lambda_{12} d \alpha_{12}+\lambda_{23} d \alpha_{23}+\lambda_{31} d \alpha_{31}\right) .
$$

(The choice of horospheres does not matter because the angle sum at the ideal vertices is constant. Also note that the lengths $\tilde{\ell}_{i j}$ of the finite edges do not appear in the equation because their dihedral angles are constant.)

The function $\hat{V}_{h}$ defined by (6-4) is

$$
\begin{aligned}
& \hat{V}_{h}\left(\lambda_{12}, \lambda_{23}, \lambda_{31}, \lambda_{1}, \lambda_{2}, \lambda_{3}\right) \\
& =\frac{1}{2}\left(\alpha_{1} \lambda_{1}+\alpha_{2} \lambda_{2}+\alpha_{3} \lambda_{3}+\alpha_{12} \lambda_{12}+\alpha_{23} \lambda_{23}+\alpha_{31} \lambda_{31}\right)+V_{h}\left(\alpha_{1}, \alpha_{2}, \alpha_{3}\right),
\end{aligned}
$$

so that

$$
d \widehat{V}_{h}=\frac{1}{2}\left(\alpha_{1} d \lambda_{1}+\alpha_{2} d \lambda_{2}+\alpha_{3} d \lambda_{3}+\alpha_{12} d \lambda_{12}+\alpha_{23} d \lambda_{23}+\alpha_{31} d \lambda_{31}\right) .
$$

From this one obtains Proposition 6.1.4 on the partial derivatives of $E_{\mathrm{T}, \Theta, \lambda}^{h}$. By extending $\widehat{V}_{h}$ using essentially the same argument as in the proof of Proposition 4.1.5, one obtains Proposition 6.1.2 on the extension of $E_{\mathrm{T}, \Theta, \lambda}^{h}$. To prove the convexity of $E_{\mathrm{T}, \Theta, \lambda}^{h}$ (Proposition 6.1.5), note that the function

$$
\widehat{V}_{h}\left(\lambda_{12}, \lambda_{23}, \lambda_{31}, \lambda_{1}, \lambda_{2}, \lambda_{3}\right)-\frac{\pi}{4}\left(\lambda_{12}+\lambda_{23}+\lambda_{31}\right)
$$

really only depends on the three parameters

$$
\begin{aligned}
& x_{1}=\frac{1}{4}\left(+\lambda_{12}-\lambda_{23}+\lambda_{31}-2 \lambda_{1}\right)=\frac{\partial V_{h}}{\partial \alpha_{1}}, \\
& x_{2}=\frac{1}{4}\left(+\lambda_{12}+\lambda_{23}-\lambda_{31}-2 \lambda_{2}\right)=\frac{\partial V_{h}}{\partial \alpha_{2}}, \\
& x_{3}=\frac{1}{4}\left(-\lambda_{12}+\lambda_{23}+\lambda_{31}-2 \lambda_{3}\right)=\frac{\partial V_{h}}{\partial \alpha_{3}},
\end{aligned}
$$


As function of these parameters, it is minus the Legendre transform of the strictly concave function $V_{h}$ :

$$
\widehat{V}_{h}-\frac{\pi}{4}\left(\lambda_{12}+\lambda_{23}+\lambda_{31}\right)=-\alpha_{1} x_{1}-\alpha_{2} x_{2}-\alpha_{3} x_{3}+V_{h}
$$

Therefore, $\widehat{V}_{h}$ is a locally strictly convex function of $x_{1}, x_{2}, x_{3}$, and hence also of $\lambda_{1}, \lambda_{2}, \lambda_{3}$, if $\lambda_{12}, \lambda_{23}, \lambda_{31}$ are considered constant. The $C^{1}$ extension of $\hat{V}_{h}$ is linear outside the domain where the triangle inequalities are satisfied, hence still convex.

Remark 6.2.2 In the same way, one can derive a theory of discrete conformal equivalence for spherical triangulations. In this case, the polyhedral building block is a tetrahedron with one finite and three ideal vertices. The functions involved in the corresponding variational principles are not convex. So in this case, the variational principles do not immediately lead to a uniqueness theorem, nor to a computational method for discrete conformal maps.

\section{Appendix A: Necessary conditions for the existence of a solu- tion of the discrete conformal mapping problems}

In this appendix, we will discuss some rather obvious and rather mild necessary conditions for the solvability of the discrete mapping problems and how they relate to the behavior of the function $E_{\mathrm{T}, \Theta, \lambda}(u)$. In short, the conditions are necessary for the problems to have a solution and sufficient to ensure that $E_{\mathrm{T}, \Theta, \lambda}(u)$ behaves "sanely", so that the following solvability alternative (see the corollary to Proposition A.2.2) holds: provided that we are able to find a minimizer of a convex function if it exists, then the variational principle allows us to either solve a discrete conformal mapping problem or to ascertain that it is not solvable.

\section{A.1 The discrete Gauss-Bonnet condition}

Condition A We have $\sum_{i \in V} \Theta_{i}=\pi|T|$.

If Problem 3.1.1 has a solution then clearly Condition A is satisfied (because the sum of angle sums around vertices equals the sum of angle sums in triangles). This is actually a discrete version of the Gauss-Bonnet formula. If we set $K_{i}=2 \pi-\Theta_{i}$ for interior vertices and $\kappa_{i}=\pi-\Theta_{i}$ for boundary vertices then Condition $\mathrm{A}$ is equivalent to

$$
\left.\sum_{i \in V_{\mathrm{int}}} K_{i}+\sum_{i \in V_{\text {bdy }}} \kappa_{i}=2 \pi(|T|-|E|+|V|)\right) .
$$


Proposition A.1.1 The function $E_{\mathrm{T}, \Theta, \lambda}(u)$ is scale-invariant, that is,

$$
E_{\mathrm{T}, \Theta, \lambda}\left(u+h 1_{V}\right)=E_{\mathrm{T}, \Theta, \lambda}(u),
$$

if and only if Condition $A$ is satisfied.

Proof Adding $h$ to every $u_{i}$ results in an added $2 h$ to every $\tilde{\lambda}_{i j}$; see (2-3). Using (4-5) and (4-6), one obtains

$$
E_{\mathrm{T}, \Theta, \lambda}\left(u+h 1_{V}\right)=E_{\mathrm{T}, \Theta, \lambda}(u)+h\left(\sum_{i \in V} \Theta_{i}-\pi|T|\right) .
$$

\section{A.2 The solvability alternative}

The following stronger Conditions B and C are also obviously necessary for the existence of a solution to Problem 3.1.1. Moreover, if a solution to the general Problem 3.1.3 exists (where $\Theta_{i}$ is prescribed only for $i \in V_{1}$ ), then positive $\Theta$-values can be assigned also to the vertices in $V_{0}$ so that Conditions B and $\mathrm{C}$ are satisfied.

Condition B There exists a system of angles $\hat{\alpha}>0$, such that

$$
\begin{aligned}
\hat{\alpha}_{j k}^{i}+\hat{\alpha}_{k i}^{j}+\hat{\alpha}_{i j}^{k}=\pi & \text { for all } i j k \in T, \\
\sum_{j k: i j k \in T} \hat{\alpha}_{j k}^{i}=\Theta_{i} & \text { for all } i \in V .
\end{aligned}
$$

Condition C If $T_{1}$ is any subset of $T$ and $V_{1} \subseteq V$ is the set of all vertices of the triangles in $T_{1}$, that is,

then

$$
V_{1}=\bigcup_{i j k \in T_{1}}\{i, j, k\}
$$

$$
\pi\left|T \backslash T_{1}\right| \geq \sum_{i \in V \backslash V_{1}} \Theta_{i},
$$

where equality holds if and only if $T_{1}=\varnothing$ or $T_{1}=T$.

Proposition A.2.1 Conditions $B$ and $C$ are equivalent.

Proof The implication 'Condition $\mathrm{B} \Rightarrow$ Condition $C$ ' is easy to see. Regarding the converse implication, Colin de Verdière proves a similar statement using the feasible flow theorem [8, Section 7]. It is straightforward to adapt his proof for Proposition A.2.1. 
Proposition A.2.2 If Condition $B$ or $C$ is satisfied (and hence both of them are), then

$$
E_{\mathrm{T}, \Theta, \lambda}(u) \rightarrow \infty \quad \text { if } \quad \max _{i \in V} u_{i}-\min _{i \in V} u_{i} \rightarrow \infty .
$$

Definition A.2.3 (Reasonably posed mapping problems) We say that Problem 3.1.1 is reasonably posed if Condition $\mathrm{B}$ or Condition $\mathrm{C}$ is satisfied (and hence both of them and Condition A are). We say that Problem 3.1.3 (where $\Theta_{i}$ is prescribed only for $i \in V_{1}$ ) is reasonably posed if positive $\Theta$-values can be assigned also to the vertices in $V_{0}$ so that Conditions B or $\mathrm{C}$ are satisfied (and hence both of them and Condition A are).

Corollary A.2.4 (Solvability alternative) If Problem 3.1.1 or Problem 3.1.3 are reasonably posed, then $E_{\mathrm{T}, \Theta, \lambda}(u)$ (maybe with some variables $u_{i}$ fixed) has a minimizer $u_{\min }$. Either $u_{\min }$ is contained in the domain where all triangle inequalities are satisfied, in which case it is unique (up to an additive constant if no variables are fixed) and corresponds to the solution of the discrete conformal mapping problem, or it lies outside that domain, in which case the corresponding discrete conformal mapping problem does not have a solution.

Proof of Proposition A.2.2 Using the (constant) angles $\hat{\alpha}$ we can rewrite the sum over vertices on the right-hand side of (4-6) as a sum over triangles:

$$
\sum_{i \in V} \Theta_{i} u_{i}=\sum_{i j k \in T}\left(\hat{\alpha}_{j k}^{i} u_{i}+\hat{\alpha}_{k i}^{j} u_{j}+\hat{\alpha}_{i j}^{k} u_{k}\right)
$$

Expressing $u$ in terms of $\tilde{\lambda}$ and $\lambda$, we obtain

$$
\begin{aligned}
\hat{\alpha}_{j k}^{i} u_{i}+\hat{\alpha}_{k i}^{j} u_{j}+\hat{\alpha}_{i j}^{k} u_{k} & \\
& =\left(\frac{\pi}{2}-\hat{\alpha}_{i j}^{k}\right)\left(\tilde{\lambda}_{i j}-\lambda_{i j}\right)+\left(\frac{\pi}{2}-\hat{\alpha}_{j k}^{i}\right)\left(\tilde{\lambda}_{j k}-\lambda_{j k}\right)+\left(\frac{\pi}{2}-\hat{\alpha}_{k i}^{j}\right)\left(\tilde{\lambda}_{k i}-\lambda_{k i}\right),
\end{aligned}
$$

so

$$
E_{\mathrm{T}, \Theta, \lambda}(u)=\sum_{i j k \in T}\left(2 f\left(\frac{\tilde{\lambda}_{i j}}{2}, \frac{\tilde{\lambda}_{j k}}{2}, \frac{\tilde{\lambda}_{k i}}{2}\right)-\hat{\alpha}_{i j}^{k} \tilde{\lambda}_{i j}-\widehat{\alpha}_{j k}^{i} \tilde{\lambda}_{j k}-\hat{\alpha}_{k i}^{j} \tilde{\lambda}_{k i}\right)+\text { const. }
$$

where here and in the following "const." stands for terms that do not depend on $u$. Using the estimate of Proposition 4.2.6 and (A-1), one obtains

$$
\begin{aligned}
E_{\mathrm{T}, \Theta, \lambda}(u) \geq & \sum_{i j k \in T}\left(\pi \max \left\{\tilde{\lambda}_{i j}, \tilde{\lambda}_{j k}, \tilde{\lambda}_{k i}\right\}-\hat{\alpha}_{i j}^{k} \tilde{\lambda}_{i j}-\hat{\alpha}_{j k}^{i} \tilde{\lambda}_{j k}-\hat{\alpha}_{k i}^{j} \tilde{\lambda}_{k i}\right)+\text { const. } \\
= & \sum_{i j k \in T}\left(\hat{\alpha}_{i j}^{k}\left(\max \{\cdots\}-\tilde{\lambda}_{i j}\right)+\hat{\alpha}_{j k}^{i}\left(\max \{\cdots\}-\tilde{\lambda}_{j k}\right)\right. \\
& \left.+\hat{\alpha}_{k i}^{j}\left(\max \{\cdots\}-\tilde{\lambda}_{k i}\right)\right)+ \text { const. }
\end{aligned}
$$




$$
\geq \min _{\substack{k \\ i j} \in}\left\{\hat{\alpha}_{i j}^{k}\right\} \sum_{i j k \in T}\left(\max \left\{\tilde{\lambda}_{i j}, \tilde{\lambda}_{j k}, \tilde{\lambda}_{k i}\right\}-\min \left\{\tilde{\lambda}_{i j}, \tilde{\lambda}_{j k}, \tilde{\lambda}_{k i}\right\}\right)+\text { const. }
$$

Now if $i j k \in T$, then $u_{i}-u_{j}=\tilde{\lambda}_{k i}-\tilde{\lambda}_{j k}-\lambda_{k i}+\lambda_{j k}$, so $\max \left\{u_{i}, u_{j}, u_{k}\right\}-\min \left\{u_{i}, u_{j}, u_{k}\right\} \leq \max \left\{\tilde{\lambda}_{i j}, \tilde{\lambda}_{j k}, \tilde{\lambda}_{k i}\right\}-\min \left\{\tilde{\lambda}_{i j}, \tilde{\lambda}_{j k}, \tilde{\lambda}_{k i}\right\}+$ const. and because the triangulated surface is connected this implies

$$
E_{\mathrm{T}, \Theta, \lambda}(u) \geq \min \left\{\hat{\alpha}_{i j}^{k}\right\}\left(\max _{i \in V} u_{i}-\min _{i \in V} u_{i}\right)+\text { const. }
$$

\section{Appendix B: The corresponding smooth conformal mapping problems and variational principles}

A natural question regarding the two variational principles for discrete conformal maps presented in Sections 4.1 and 4.3 is: "What are the corresponding variational principles in the classical smooth theory of conformal maps?" In fact, even the question "What exactly are the corresponding smooth mapping problems?" deserves a comment. For the second variational principle it is not even obvious how the variables - triangle angles - translate to the smooth theory.

\section{B.1 Background: Curvature, unit vector fields and conformal metrics}

Before we address these questions in Sections B.2 and B.3, we outline some classical background material from the differential geometry of surfaces. The purpose is twofold: first, to fix notation; second, our exposition takes a particular point of view, focusing on unit vector fields, which prepares the discussion of the second variational principle in Section B.3.

Let $M$ be a smooth oriented surface, possibly with boundary, equipped with a Riemannian metric $g$ and the induced Levi-Civita connection $\nabla$. The Riemannian metric and orientation induce a $90^{\circ}$-rotation tensor

$$
J: T M \rightarrow T M
$$

and an area $2-$ form

$$
\sigma=g(J \cdot, \cdot)
$$

A unit vector field on $M$ is a tangent vector field $Y$ with $g(Y, Y)=1$. Of course, the existence of a unit vector field imposes restrictions on the topology of $M$. In any 
case, unit vector fields exist locally, so purely local considerations remain valid for arbitrary $M$. The Gauss curvature $K \in C^{\infty}(M)$ is defined by the equation

$$
K=-g(R(Y, J Y) Y, J Y),
$$

where $Y$ is any unit vector field, and $R$ denotes the Riemann curvature tensor,

$$
R(X, Y) Z=\nabla_{X} \nabla_{Y} Z-\nabla_{Y} \nabla_{X} Z-\nabla_{[X, Y]} Z .
$$

The curvature 2-form is defined by

$$
\Omega=K \sigma .
$$

For a unit vector field $Y$, we define the rotation $1-$ form $\rho_{Y}$ by

$$
\rho_{Y}(X)=g\left(\nabla_{X} Y, J Y\right) .
$$

Proposition B.1.1 For any unit vector field $Y$,

$$
d \rho_{Y}=-\Omega \text {. }
$$

Proof The claim follows from the definition of the Riemann curvature tensor (B-1), by a straightforward calculation:

$$
\begin{aligned}
d \rho_{Y}(Y, J Y)= & Y \cdot g\left(\nabla_{J Y} Y, J Y\right)-(J Y) \cdot g\left(\nabla_{Y} Y, J Y\right)-g\left(\nabla_{[Y, J Y]} Y, J Y\right) \\
= & g\left(\nabla_{Y} \nabla_{J Y} Y, J Y\right)+\underbrace{g\left(\nabla_{J Y} Y, \nabla_{Y} J Y\right)}_{=0} \\
& \quad-g\left(\nabla_{J Y} \nabla_{Y} Y, J Y\right)-\underbrace{g\left(\nabla_{Y} Y, \nabla_{J Y} J Y\right)}_{=0}-g\left(\nabla_{[Y, J Y]} Y, J Y\right) \\
= & g(R(Y, J Y) Y, J Y)=-K .
\end{aligned}
$$

We have used that $\nabla_{V} Y \perp Y$ and $\nabla_{V} J Y \perp J Y$ for any vector field $V$, so that, because $M$ is two-dimensional, $g\left(\nabla_{V} Y, \nabla_{W} J Y\right)=0$ for any $V, W$.

Now consider a conformal change of metric with conformal factor $e^{u}$ determined by (1-1). Note that a conformal change of metric is also characterized by the fact that the $90^{\circ}$-rotation with respect to the new metric $\widetilde{g}$ is the same tensor $J$. The Levi-Civita connection $\tilde{\nabla}$ of $\widetilde{g}$ is related to the Levi-Civita connection $\nabla$ of $g$ by

$$
\widetilde{\nabla}_{X} Z=\nabla_{X} Z+g(X, G) Z+g(Z, G) X-g(X, Z) G,
$$

where $G=\operatorname{grad}_{g} u$, that is, $d u=g(G, \cdot)$. A unit vector field $Y$ with respect to $g$ naturally determines a unit vector field

$$
\tilde{Y}=e^{-u} Y
$$


with respect to $\widetilde{g}$. Its rotation $1-$ form is

$$
\tilde{\rho}_{\tilde{Y}}(X)=\tilde{g}\left(\tilde{\nabla}_{X} \tilde{Y}, J \tilde{Y}\right) .
$$

Proposition B.1.2 The rotation $1-$ forms $\rho_{Y}, \tilde{\rho}_{\tilde{Y}}$ are related by

$$
\tilde{\rho}_{\tilde{Y}}=\rho_{Y}+* d u,
$$

where $*$ denotes the Hodge star operator for $g$.

The Hodge star operator $*$ maps a 1 -form $\omega$ to the 1 -form $* \omega=-\omega(J \cdot)$. It also maps a function ( 0 -form) $f$ to the 2 -form $* f=f \sigma$ and vice versa, $* f \sigma=f$. Note that on a 2-dimensional manifold, the action of the Hodge star operator on 1-forms depends only on the conformal class of the metric.

Proof of Proposition B.1.2 By the product rule,

$$
\tilde{\nabla}_{X} \tilde{Y}=e^{-u}\left(-d u(X) Y+\tilde{\nabla}_{X} Y\right),
$$

so $\tilde{\rho}_{\widetilde{Y}}(X)=g\left(\widetilde{\nabla}_{X} Y, J Y\right)$. Using (B-2) one obtains

$$
\tilde{\rho}_{\widetilde{Y}}(X)=\rho_{Y}(X)+g(Y, G) g(X, J Y)-g(X, Y) g(G, J Y) .
$$

Finally, since $J$ is skew, $J^{2}=-1$, and $(Y, J Y)$ is an orthonormal frame,

$$
\begin{aligned}
g(Y, G) g(X, J Y)-g(X, Y) g(G, J Y) & =-g(G, Y) g(Y, J X)-g(G, J Y) g(J Y, J X) \\
& =-g(G, J X)=-d u(J X)=* d u(X) .
\end{aligned}
$$

This completes the proof.

As a corollary of Propositions B.1.1 and B.1.2, we obtain the equation relating the curvature 2-forms of $g$ and $\tilde{g}$,

$$
\widetilde{\Omega}=\Omega-d * d u,
$$

and hence Liouville's equation for the curvatures,

$$
e^{2 u} \tilde{K}=K+\Delta u,
$$

where $\Delta$ is the Laplace-Beltrami operator with respect to $g$,

$$
\Delta f=-* d * d f .
$$

(We use the sign convention for the Laplace operator that renders it positive semidefinite.) 


\section{B.2 Smooth mapping problems and the first variational principle}

Which problems in the smooth theory are analogous to the discrete conformal mapping problems discussed in this paper? There are two fairly obvious candidates:

Problem B.2.1 Given $(M, g)$ and the function $\tilde{K}$, find a conformally equivalent Riemannian metric $\tilde{g}=e^{2 u} g$ with curvature $\widetilde{K}$.

This amounts to solving Liouville's equation (B-4) for $u$.

Problem B.2.2 Given $(M, g)$ and the 2 -form $\widetilde{\Omega}$, find a conformally equivalent Riemannian metric $\widetilde{g}=e^{2 u} g$ with curvature 2 -form $\tilde{\Omega}$.

Prescribing the target curvature 2-form is equivalent to prescribing $e^{2 u} \tilde{K}$ instead of the target curvature $\widetilde{K}$. Problem B.2.2 amounts to solving (B-3), which is equivalent to Poisson's equation

$$
\Delta u=f
$$

with right-hand side $f=* \widetilde{\Omega}-K$.

For both problems, one may prescribe either $u$ on the boundary (Dirichlet conditions) or $\left.* d u\right|_{T \partial M}$ with $\int_{\partial M} * d u=-\int_{M}(\widetilde{\Omega}-\Omega)$ (Neumann conditions).

Both Liouville's equation (B-4) and the Poisson equation (B-3) are variational, with the respective functionals

$$
E_{A}(u)=\int_{M}\left(\frac{1}{2} d u \wedge * d u-\left(\frac{1}{2} e^{2 u} \tilde{K}-u K\right) \sigma\right)
$$

for Liouville's equation, where $\widetilde{K}$ and $K$ are fixed given functions on $M$, and

$$
E_{B}(u)=\int_{M}\left(\frac{1}{2} d u \wedge * d u-u(\widetilde{\Omega}-\Omega)\right)
$$

for (B-3), where $\widetilde{\Omega}$ and $\Omega$ are fixed given 2-forms on $M$.

Question Which of the two candidates, Problem B.2.1 or Problem B.2.2, is the smooth version of the discrete mapping problems described in Section 3?

Answer Comparing the scaling behavior shows that the discrete mapping problems are discretization of Problem B.2.2 and not of Problem B.2.1. The function $E_{\mathrm{T}, \Theta, \lambda}$ of the first variational principle corresponds to $E_{B}$ and not to $E_{A}$.

Indeed, although we did denote the angle defect $2 \pi-\Theta_{i}$ at a vertex $i$ of a triangulation by $K_{i}$ in Appendix A, this angle defect is a discretization of the curvature 2 -form $\Omega$ 
and not of the Gauss curvature $K$, the latter being an angle defect per surface area. The difference manifests itself in the scaling behavior: the angle defects at vertices and the curvature 2-form remain invariant if lengths are scaled by a constant factor. The Gauss curvature, on the other hand, is inversely proportional to the square of the scale factor. Thus, prescribing the angle defects $K_{i}$ at the vertices of a triangulation corresponds to prescribing the curvature 2 -form $\Omega$ of a smooth surface, as in Problem B.2.2, and not the Gauss curvature $K$, as in Problem B.2.1.

Remark B.2.3 For $\widetilde{K}=0$ and $\widetilde{\Omega}=0$ there is no difference between Problems B.2.1 and B.2.2, and $E_{A}=E_{B}$.

Remark B.2.4 Consider the gradient flow of the discrete functional $E_{\mathrm{T}, \Theta, \lambda}$. For a closed triangulated surface with prescribed angle sum $\Theta_{i}=2 \pi$ for all vertices, this gradient flow is equal to

$$
\dot{u}_{i}(t)=-K_{i}(t)
$$

where $K_{i}(t)$ is the angle defect around vertex $i$ at time $t$. At first glance, this looks like a discrete version of the Ricci flow for surfaces [24]. For surfaces, Ricci flow is the same as Yamabe flow because the Ricci tensor is proportional to the Riemannian metric. The Riemannian metric evolves conformally, $g_{t}=e^{2 u_{t}} g_{0}$, according to the law

$$
\dot{u}_{t}=-K_{t},
$$

where $K_{t}$ is the Gauss curvature at time $t$.

However, the above comparison of the scaling behaviors of angle defect $K_{i}$ and Gauss curvature $K$ shows that (B-7) is not a discretization of (B-8). In fact, the flow (B-7) is a discretization of the gradient flow of $E_{B}$,

$$
\dot{u}_{t}=-e^{2 u_{t}} K_{t},
$$

which is a curvature flow for surfaces that is different from the Ricci/Yamabe flow.

The same scaling argument applies to other flows that have mistakenly been tagged combinatorial or discrete Ricci flow $[7 ; 16]$.

\section{B.3 The second variational principle and harmonic unit vector fields}

The variables of the second variational principle (Section 4.3) are assignments of new angles in a euclidean triangulation. How do these variables translate to smooth manifolds? Consider an angle vector $\alpha \in\left(\mathbb{R}_{>0}\right)^{A_{\top}}$ that assigns angle values $\alpha_{j k}^{i}>0$ 
to the corners $\underset{j k}{i} \in A_{\mathrm{T}}$ of the triangles in such a way that the sum is $\pi$ in each triangle. While such an angle assignment fixes the shape of each triangle (up to similarity), a consistent assignment of edge lengths is generally not possible. The assigned angles do, however, induce a sensible definition of parallel transport of unit vectors from edge to edge: the unit vector that makes an angle $\beta_{i j}$ with the directed edge $i j$ in triangle $i j k$ is transported to the unit vector that makes an angle $\beta_{j k}=\beta_{i j}+\alpha_{k i}^{j}-\pi$ with edge $j k$ (see Figure 21).

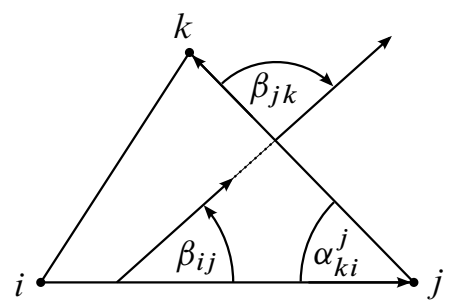

Figure 21: Parallel transport of directions from edge to edge

Thus, an angle assignment $\alpha \in\left(\mathbb{R}_{>0}\right)^{A_{\top}}$ in a euclidean triangulation corresponds to a connection of the unit tangent bundle $T_{1} M$ of the smooth surface $M$.

For simplicity, our discussion of the second variational principle will focus on the special case when the triangulation is topologically a closed disk and the prescribed angle sums at interior vertices are $2 \pi$. In the smooth setting, angle assignments that sum to $\pi$ in each triangle and to $2 \pi$ around each vertex correspond to flat connections of the unit tangent bundle. Since the surface is assumed to be simply connected, for any such flat connection there exists a parallel unit vector field and this is unique up to rotation by a constant angle. Conversely, any unit vector field is parallel for a unique flat connection. Thus, this special case allows a more intuitive treatment involving unit vector fields and rotation 1 -forms instead of connections and connection 1 -forms. At the end of this section, we will indicate how to treat the general case.

So assume for now that $M$ is diffeomorphic to a closed disk and consider Problem B.2.2 with $\widetilde{\Omega}=0$. That is, we are looking for a conformally equivalent flat metric. The Dirichlet energy of a unit vector field $Y$ is

$$
S(Y)=\frac{1}{2} \int_{M} \rho_{Y} \wedge * \rho_{Y}
$$

Critical points of this Dirichlet energy are the harmonic sections of the unit tangent bundle. Admissible variations are within the space of unit vector fields, fixing the values on the boundary. 
Proposition B.3.1 (First variation) Let $Y_{t}$ be a variation of the unit vector field $Y=Y_{0}$ with

$$
\left.\frac{d}{d t}\right|_{t=0} Y_{t}=h J Y
$$

where $h \in C^{\infty}(M)$. Then

$$
\left.\frac{d}{d t}\right|_{t=0} S\left(Y_{t}\right)=-\int_{M} h d * \rho_{Y}+\int_{\partial M} h * \rho_{Y} .
$$

Proof This follows from the equation $\left.\frac{d}{d t}\right|_{t=0} \rho_{Y_{t}}=d h$.

Corollary B.3.2 A unit vector field $Y$ is a critical point of $S$ under variations that fix $Y$ on the boundary $\partial M$ if and only if

$$
d * \rho_{Y}=0 .
$$

It is also a critical point of $S$ under arbitrary variations if and only if, additionally,

$$
\left.* \rho_{Y}\right|_{T \partial M}=0 .
$$

Loosely speaking, the following proposition says that straightest unit vector fields with respect to $g$ are parallel with respect to a conformally equivalent flat metric $\tilde{g}$ with trivial global holonomy.

Proposition B.3.3 (Smooth version of the second variational principle) Suppose the unit vector field $Y$ is a critical point of $S$ under variations that fix $Y$ on the boundary. Define the function $u$ up to an additive constant by

$$
d u=* \rho_{Y} .
$$

(This is possible since $* \rho_{Y}$ is closed by the above corollary and we assumed that $M$ was diffeomorphic to a disk.) Let $\widetilde{g}=e^{2 u} g$. Then we have the following.

(i) The unit vector field $\tilde{Y}=e^{-u} Y$ is parallel with respect to $\widetilde{g}$, so $\widetilde{g}$ is flat.

(ii) The geodesic curvature of the boundary $\partial M$ with respect to $\tilde{g}$ is

$$
\tilde{\kappa}=\kappa-\rho_{Y}(T),
$$

where $\kappa$ is the geodesic curvature with respect to $g$ and $T$ is the positively oriented unit tangent vector field to $\partial M$.

(iii) If $Y$ is also a critical point of $S$ under arbitrary variations, then $\left.u\right|_{\partial M}$ is constant. 
Proof Since $* d u=-\rho_{Y}$, the rotation form of $\tilde{\rho} \widetilde{Y}$ vanishes by Proposition B.1.2. This implies (i). The geodesic boundary curvatures are $\kappa=\rho_{T}(T)$ and $\widetilde{\kappa}=\tilde{\rho} \widetilde{T}(\widetilde{T})$. (Locally extend the unit vector field $T$ inwards from the boundary.) Again by Proposition B.1.2, this implies (ii). Finally, (iii) follows immediately from (B-10).

In the general case, $M$ is not restricted to be diffeomorphic to a closed disk and one is looking for a conformally equivalent metric $\widetilde{g}$ with prescribed curvature 2 -form $\widetilde{\Omega}$. To treat this case variationally, consider the functional

$$
S(\rho)=\frac{1}{2} \int_{M} \rho \wedge * \rho
$$

on the affine space of 1 -forms $\rho$ satisfying $d \rho=\widetilde{\Omega}-\Omega$. We leave the details to the reader, not because they are tedious but because they are interesting. The critical points correspond to conformally equivalent similarity structures, that is, "metrics" which may have global scaling holonomy. (Compare the remark at the end of Section 4.3.)

\section{Appendix C: Relation to circle patterns}

\section{C.1 Two variational principles for circle patterns}

While the discrete conformal mapping problems essentially ask for ideal hyperbolic polyhedra with prescribed metric, the circle pattern problem below asks for an ideal polyhedron with prescribed dihedral angles. Rivin's variational principle for this type of problem [34] is very similar to our second variational principle for discrete conformal maps. The function is essentially the same, only the constraints placed on the angle assignments are stronger. Also, the first variational principle needs only a slight modification to become a variational principle for circle patterns.

Problem C.1.1 (Circle pattern problem) Given a surface triangulation $T$ and a function $\Phi \in(0, \pi]^{E}$, find a discrete metric $\tilde{\ell}$ so that the euclidean triangulation $(T, \tilde{\ell})$ has circumcircle intersection angles $\Phi_{i j}$ as shown in Figure 22.

Proposition C.1.2 (Rivin [34]) The angles $\widetilde{\alpha} \in \mathbb{R}^{A}$ are the angles of a euclidean triangulation $(\mathrm{T}, \tilde{\ell})$ that solves Problem C.1.1 if and only if $S_{\mathrm{T}, \lambda}(\widetilde{\alpha})$ is the maximum of $S_{\mathrm{T}, \lambda}$ on the set of all $\alpha \in \mathbb{R}^{A}$ that satisfy:

(i) $\alpha>0$.

(ii) $\alpha_{i j}^{k}+\alpha_{j k}^{i}+\alpha_{k i}^{j}=\pi$ for all triangles $i j k \in T$.

(iii) $\alpha_{i j}^{k}+\alpha_{j k}^{l}=\Phi_{i j}$ for all interior edges $i j \in E_{\mathrm{int}}$.

(iv) $\alpha_{i j}^{k}=\Phi_{i j}$ for all boundary edges $i j \in E_{\mathrm{bdy}}$. 


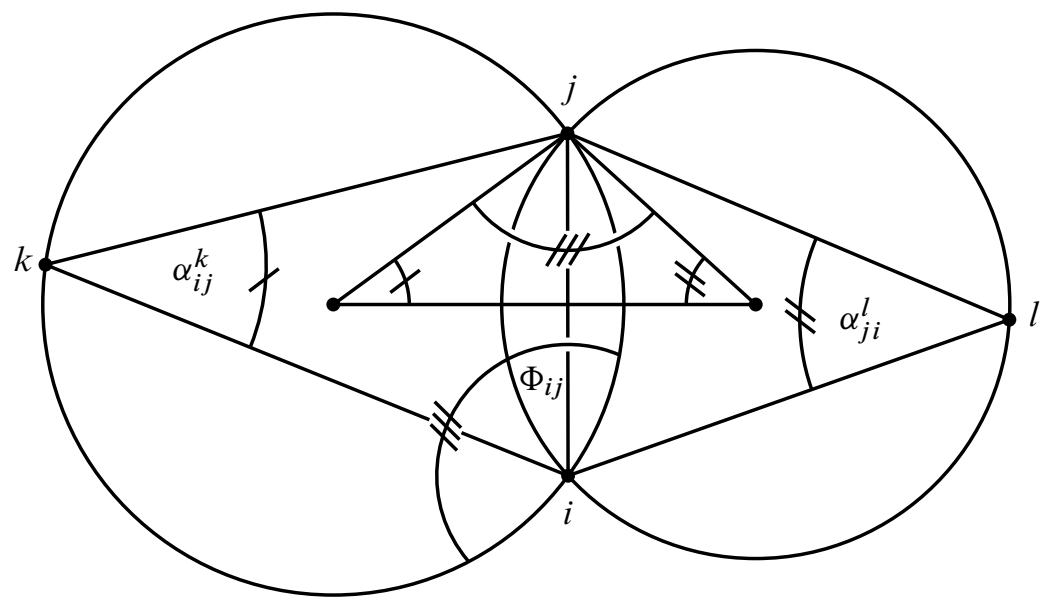

Figure 22: Circumcircle intersection angles $\Phi_{i j}=\alpha_{i j}^{k}+\alpha_{j i}^{l}$ : for a boundary edge $i j \in E_{\mathrm{bdy}}$, define $\Phi_{i j}=\alpha_{i j}^{k}$.

Due to conditions (iii) and (iv), the choice of the parameter $\lambda \in \mathbb{R}^{E}$ of $S_{\mathrm{T}, \lambda}$ does not matter because

$$
S_{\mathrm{T}, \lambda}(\alpha)=S_{\mathrm{T}, 0}(\alpha)+\sum_{i j \in E} \Phi_{i j} \lambda_{i j}
$$

So in connection with circle patterns, it makes sense to consider only

$$
S_{\mathrm{T}, 0}(\alpha)=\sum \pi\left(\alpha_{i j}^{k}\right) \text {. }
$$

Now consider the first variational principle for discrete conformal maps. For $\Phi \in \mathbb{R}^{E}$, $\Theta \in \mathbb{R}^{V}$ define

$$
\begin{aligned}
\mathcal{E}_{\mathrm{T}, \Phi, \Theta}: \mathbb{R}^{E} \times \mathbb{R}^{V} \rightarrow \mathbb{R} & \\
\mathcal{E}_{\mathrm{T}, \Phi, \Theta}(\lambda, u)=\sum_{i j k \in T} 2 \widehat{V}\left(\lambda_{i j}, \lambda_{j k}, \lambda_{k i},\right. & \left.-u_{i},-u_{j},-u_{k}\right) \\
& -\sum_{i j \in E} \Phi_{i j} \lambda_{i j}+\sum_{i \in V} \Theta_{i} u_{i}
\end{aligned}
$$

compare (5-6). If $\Phi$ is defined by (5-7), then

$$
\mathcal{E}_{\mathrm{T}, \Phi, \Theta}(\lambda, u)=E_{\mathrm{T}, \Theta, \lambda}(u) .
$$

So if we fix $\lambda$ and vary $u$, then we obtain the first variational principle for discrete conformal maps. If, on the other hand, we fix $u$ and vary $\lambda$, then we obtain a variational principle for circle patterns (see Proposition C.1.3 below). Interpret the circumcircles and triangle sides as hyperbolic planes in the half-plane model. Then, using (5-5) and 
the fact that opposite dihedral angles in an ideal tetrahedron are equal, one gets for an interior edge $i j \in E_{\text {int }}$ that

$$
\frac{\partial}{\partial \lambda_{i j}} \mathcal{E}_{\mathrm{T}, \Phi, \Theta}=\tilde{\alpha}_{i j}^{k}+\widetilde{\alpha}_{j i}^{l}-\Phi_{i j}
$$

where $\tilde{\alpha}$ are the angles in the euclidean triangulation $(\mathrm{T}, \tilde{\ell})$ with $\tilde{\ell}$ determined by $(2-1)$ and (2-2). (Here we assume that $\tilde{\ell}$ satisfies the triangle inequalities. Otherwise the angles are 0 or $\pi$ as stipulated in Proposition 4.2.5.) In the same way, one gets for a boundary edge $i j$

$$
\frac{\partial}{\partial \lambda_{i j}} \mathcal{E}_{\mathrm{T}, \Phi, \Theta}=\tilde{\alpha}_{i j}^{k}-\Phi_{i j}
$$

implying the following variational principle for circle patterns. (Note that $\mathcal{E}_{\mathrm{T}, \Phi, \Theta}(\lambda, u)$ is also convex if we fix $u$ and consider $\lambda$ as variables.)

Proposition C.1.3 The function $\tilde{\ell} \in \mathbb{R}_{>0}^{E}$ defined in terms of $\lambda$ and $u$ by (2-1) and (2-2) is a solution of Problem C.1.1 if and only if $\tilde{\ell}$ satisfies all triangle inequalities and $\mathcal{E}_{\mathrm{T}, \theta, \Theta}(\lambda, u)$ is the minimum of the function $\lambda \mapsto \mathcal{E}_{\mathrm{T}, \theta, \Theta}(\lambda, u)$. That is, $u$ is arbitrary but constant. (Without loss of generality one could fix $u=0$.)

\section{C.2 Discrete conformal equivalence for circular polyhedral surfaces}

In this section, we generalize the notion of discrete conformal equivalence from surfaces composed of triangles to surfaces composed of polygons inscribed in circles. The variational principle described below is like a mixture of the first variational principle for discrete conformal maps of Proposition 4.1.3 and the variational principle for circle patterns of Proposition C.1.3.

An (abstract) polyhedral surface is a surface that is a CW-complex. A euclidean polyhedral surface is a polyhedral surface obtained by gluing euclidean polygons edge-to-edge. If all of the polygons have a circumscribed circle, we speak of a (euclidean) circular polyhedral surface. A circular polyhedral surface is determined by the polyhedral surface $\mathrm{P}$ and the function $\ell \in\left(\mathbb{R}_{>0}\right){ }^{E_{\mathrm{P}}}$ that assigns to each edge its length. Conversely, a function $\ell \in\left(\mathbb{R}_{>0}\right)^{E_{\mathrm{P}}}$ defines a circular polyhedral surface if and only if it satisfies the "polygonal inequalities": in each polygon, the length of any edge is smaller then the sum of lengths of the other edges. If $\ell$ satisfies these conditions, we denote the resulting circular polyhedral surface by $(P, \ell)$.

Definition C.2.1 Two circular polyhedral surfaces, $(\mathrm{P}, \ell)$ and $(\mathrm{P}, \tilde{\ell})$, are discretely conformally equivalent if $\ell$ and $\tilde{\ell}$ are related by (2-1) for some function $u \in \mathbb{R}^{V_{\mathrm{P}}}$. 
To solve the discrete conformal mapping problems for circular polyhedral surfaces that are analogous to those described in Section 3, proceed as follows: first triangulate the nontriangular faces of the given circular polyhedral surface $(\mathrm{P}, \ell)$ to obtain a euclidean triangulation $(\mathrm{T}, \hat{\ell})$ (where $\hat{\ell}: E_{\mathrm{T}} \rightarrow \mathbb{R}_{>0},\left.\hat{\ell}\right|_{E_{\mathrm{P}}}=\ell$ ). Then define $\Phi$ by (5-7) and minimize $\mathcal{E}_{\mathrm{T}, \Phi, \Theta}(\lambda, u)$, where $\lambda_{i j}=2 \log \hat{\ell}_{i j}$ is held fixed if $i j \in E_{\mathrm{P}}$ and considered a variable if $E_{\mathrm{T}} \backslash E_{\mathrm{P}}$, and the $u_{i}$ are variables or fixed depending on the mapping problem, as in the case of triangulations. If $\tilde{\ell}$ determined by (2-1) and (2-2) for the minimizing $(\lambda, u)$ satisfies the triangle inequalities, it is a solution of the mapping problem.

Note that the values $\hat{\ell}_{i j}$ for edges $i j \in E_{\mathrm{T}} \backslash E_{\mathrm{P}}$ do not enter because the corresponding $\lambda_{i j}$ are variables.

\section{C.3 Discrete circle domains}

A domain in the Riemann sphere $\widehat{\mathbb{C}}$ is called a circle domain if every boundary component is either a point or a circle. Koebe conjectured that every domain in $\mathbb{C}$ is conformally equivalent to a circle domain. For a simply connected domain, this is just the Riemann mapping theorem. Koebe himself proved the conjecture for finitely connected domains, and after various generalizations by several other people, He and Schramm gave a proof for domains with at most countably many boundary components [17]. (Their proof is based on circle packings.)

The method for mapping to the sphere described in Section 3.2 works (mutatis mutandis) also for the circular polyhedral surfaces discussed in the previous section. This allows us to map euclidean triangulations to "discrete circle domains", that is, domains in the plane that are bounded by circular polygons.

Suppose $(T, \ell)$ is a euclidean triangulation that is topologically a disc with holes. To map $(T, \ell)$ to a discrete circle domain, simply fill the holes by attaching a face to each boundary polygon and map the resulting circular polyhedral surface to the sphere.

Note that for a topological disk with 0 holes, we recover in a different guise the procedure for mapping to a disk that was described in Section 3.3.

Acknowledgments We are grateful to Richard Kenyon and Günter Ziegler, who pointed out the connection with amoebas, to Ulrich Bauer, Felix Günther, Mathias Oster and Hana Kouřimská, who spotted typos and instances of questionable style in earlier versions of this paper, and to the referee whose comments helped us improve this paper still further. Any remaining mistakes and shortcomings are our responsibility.

Stefan Sechelmann produced Figure 18 using software written by himself in Java together with the Java-based 3D visualization package $j$ Reality. 
The other figures of discrete conformal maps were made with Blender and Python scripts written by the third author, which rely on other libraries to do the real work: the GNU Scientific Library, providing an implementation of Clausen's integral, the convex optimization library CVXOPT by M Andersen, J Dahl and L Vandenberghe, and J Shewchuk's mesh generator Triangle.

This research was supported by SFB/TR 109 "Discretization in Geometry and Dynamics", the DFG Research Unit "Polyhedral surfaces" and by the DFG Research Center Matheon.

\section{References}

[1] A D Alexandrov, Convex polyhedra, Springer, Berlin (2005) MR2127379

[2] E M Andreev, On convex polyhedra in Lobačevskii spaces, Math. USSR-Sb. 10 (1970) 413-440

[3] E M Andreev, On convex polyhedra of finite volume in Lobačevskii space, Math. USSR-Sb. 12 (1970) 255-259

[4] A I Bobenko, T Hoffmann, B A Springborn, Minimal surfaces from circle patterns: geometry from combinatorics, Ann. of Math. 164 (2006) 231-264 MR2233848

[5] A I Bobenko, I Izmestiev, Alexandrov's theorem, weighted Delaunay triangulations and mixed volumes, Ann. Inst. Fourier (Grenoble) 58 (2008) 447-505 MR2410380

[6] A I Bobenko, B A Springborn, Variational principles for circle patterns and Koebe's theorem, Trans. Amer. Math. Soc. 356 (2004) 659-689 MR2022715

[7] B Chow, F Luo, Combinatorial Ricci flows on surfaces, J. Differential Geom. 63 (2003) 97-129 MR2015261

[8] Y Colin de Verdière, Un principe variationnel pour les empilements de cercles, Invent. Math. 104 (1991) 655-669 MR1106755

[9] J Dai, X D Gu, F Luo, Variational principles for discrete surfaces, Adv. Lect. Math. 4, International Press, Somerville, MA (2008) MR2439807

[10] R J Duffin, Distributed and lumped networks, J. Math. Mech. 8 (1959) 793-826 MR0106032

[11] D B A Epstein, R C Penner, Euclidean decompositions of noncompact hyperbolic manifolds, J. Differential Geom. 27 (1988) 67-80 MR918457

[12] V V Fock, Dual Teichmüller spaces arXiv:dg-ga/9702018

[13] D Futer, F Guéritaud, From angled triangulations to hyperbolic structures, from: "Interactions between hyperbolic geometry, quantum topology and number theory", (A Champanerkar, O Dasbach, E Kalfagianni, I Kofman, W Neumann, N Stoltzfus, editors), Contemp. Math. 541, Amer. Math. Soc. (2011) 159-182 MR2796632 
[14] M von Gagern, J Richter-Gebert, Hyperbolization of Euclidean ornaments, Electron. J. Combin. 16 (2009) MR2515775

[15] I M Gelfand, M M Kapranov, A V Zelevinsky, Discriminants, resultants and multidimensional determinants, Birkhäuser, Boston, MA (1994) MR1264417

[16] X D Gu, S-T Yau, Computational conformal geometry, Adv. Lect. Math. 3, International Press, Somerville, MA (2008) MR2439718

[17] Z-X He, O Schramm, Fixed points, Koebe uniformization and circle packings, Ann. of Math. 137 (1993) 369-406 MR1207210

[18] R Kenyon, Lectures on dimers, from: "Statistical mechanics", (S Sheffield, T Spencer, editors), IAS/Park City Math. Ser. 16, Amer. Math. Soc. (2009) 191-230 MR2523460

[19] R Kenyon, A Okounkov, Planar dimers and Harnack curves, Duke Math. J. 131 (2006) 499-524 MR2219249

[20] R Kenyon, A Okounkov, S Sheffield, Dimers and amoebae, Ann. of Math. 163 (2006) 1019-1056 MR2215138

[21] P Koebe, Kontaktprobleme der konformen Abbildung, Ber. Sächs. Akad. Wiss. Leipzig, Math.-phys. K1. 88 (1936) 141-164

[22] G Leibon, Characterizing the Delaunay decompositions of compact hyperbolic surfaces, Geom. Topol. 6 (2002) 361-391 MR1914573

[23] L Lewin, Polylogarithms and associated functions, North-Holland, New York (1981) MR618278

[24] F Luo, Combinatorial Yamabe flow on surfaces, Commun. Contemp. Math. 6 (2004) 765-780 MR2100762

[25] F Luo, Rigidity of polyhedral surfaces, from: "Fourth International Congress of Chinese Mathematicians”, (L Ji, K Liu, L Yang, S-T Yau, editors), AMS/IP Stud. Adv. Math. 48, Amer. Math. Soc. (2010) 201-217 MR2744222

[26] G Mikhalkin, Amoebas of algebraic varieties and tropical geometry, from: "Different faces of geometry", (S Donaldson, Y Eliashberg, M Gromov, editors), Int. Math. Ser. 3, Kluwer/Plenum, New York (2004) 257-300 MR2102998

[27] J Milnor, Hyperbolic geometry: The first 150 years, Bull. Amer. Math. Soc. 6 (1982) 9-24 MR634431

[28] J Milnor, How to compute volume in hyperbolic space, from: "Collected papers, Vol. 1”, Publish or Perish, Houston, TX (1994) 189-212 MR1277810

[29] J Milnor, The Schläfli differential equality, from: "Collected papers, Vol. 1", Publish or Perish, Houston, TX (1994) 281-295 MR1277810

[30] A Papadopoulos (editor), Handbook of Teichmüller theory, Vol. I, IRMA Lectures Math. Theor. Physics 11, Euro. Math. Soc., Zürich (2007) MR2284826 
[31] M Passare, H Rullgård, Amoebas, Monge-Ampère measures and triangulations of the Newton polytope, Duke Math. J. 121 (2004) 481-507 MR2040284

[32] R C Penner, The decorated Teichmüller space of punctured surfaces, Comm. Math. Phys. 113 (1987) 299-339 MR919235

[33] U Pinkall, K Polthier, Computing discrete minimal surfaces and their conjugates, Experiment. Math. 2 (1993) 15-36 MR1246481

[34] I Rivin, Euclidean structures on simplicial surfaces and hyperbolic volume, Ann. of Math. 139 (1994) 553-580 MR1283870

[35] I Rivin, Intrinsic geometry of convex ideal polyhedra in hyperbolic 3-space, from: "Analysis, algebra, and computers in mathematical research", (M Gyllenberg, editor), Lecture Notes Pure Appl. Math. 156, Dekker, New York (1994) 275-291 MR1280952

[36] I Rivin, Combinatorial optimization in geometry, Adv. in Appl. Math. 31 (2003) 242271 MR1985831

[37] M Roček, R M Williams, The quantization of Regge calculus, Z. Phys. C 21 (1984) 371-381 MR734698

[38] B Rodin, D Sullivan, The convergence of circle packings to the Riemann mapping, J. Differential Geom. 26 (1987) 349-360 MR906396

[39] R K W Roeder, J H Hubbard, W D Dunbar, Andreev's theorem on hyperbolic polyhedra, Ann. Inst. Fourier (Grenoble) 57 (2007) 825-882 MR2336832

[40] B Springborn, P Schröder, U Pinkall, Conformal equivalence of triangle meshes, ACM Trans. Graph. 27 (2008) 11

[41] K Stephenson, Introduction to circle packing, Cambridge Univ. Press (2005) MR2131318

[42] W P Thurston, The geometry and topology of three-manifolds, lecture notes (1979) Available at http://msri.org/publications/books/gt3m/

[43] W P Thurston, Minimal stretch maps between hyperbolic surfaces (1998) arXiv: math/9801039

[44] M Troyanov, Les surfaces euclidiennes à singularités coniques, Enseign. Math. 32 (1986) 79-94 MR850552

Technische Universität Berlin, Institut für Mathematik

Strasse des 17. Juni 136, 10623 Berlin, Germany

bobenko@math.tu-berlin.de, pinkall@math.tu-berlin.de, boris.springborn@tu-berlin.de

http://page.math.tu-berlin.de/ bobenko, http://page.math.tu-berlin.de/ pinkall, http://page.math.tu-berlin.de/ springb

Proposed: David Gabai

Seconded: Danny Calegari, Jean-Pierre Otal

Received: 16 September 2013

Revised: 4 August 2014 
